\title{
Multi-omic brain and behavioral correlates of cell-free fetal DNA methylation in macaque maternal obesity models
}

Authors: Benjamin I. Laufer ${ }^{1,2,3}$, Yu Hasegawa ${ }^{4, \ddagger}$, Zhichao Zhang $^{4, \neq}$, Casey E. Hogrefe ${ }^{5}$, Laura A. Del Rosso ${ }^{5}$, Lori Haapanan ${ }^{5}$, Hyeyeon Hwang ${ }^{1,2,3}$, Melissa D. Bauman ${ }^{3,5,6}$, Judy A. Van de Water ${ }^{7}$, Ameer Y. Taha4, Carolyn M. Slupsky,8, Mari S. Golub5 , John P. Capitanio ${ }^{5,9}$, Catherine A. VandeVoort ${ }^{5,10}$ Cheryl K. Walker ${ }^{3,5,10,11}$, Janine M. LaSalle ${ }^{1,2,3,11, *}$

Affiliations:

${ }^{1}$ Department of Medical Microbiology and Immunology, School of Medicine, University of California Davis, Davis, CA, 95616, USA

2 UC Davis Genome Center, University of California, Davis, CA, 95616, USA

${ }^{3}$ MIND Institute, School of Medicine, University of California Davis, Sacramento, CA, 95817, USA

${ }^{4}$ Department of Food Science and Technology, University of California Davis, Davis, CA, 95616, USA

${ }^{5}$ California National Primate Research Center, University of California Davis, Davis, CA, 95616, USA

${ }^{6}$ Department of Psychiatry and Behavioral Sciences, School of Medicine, University of California Davis, Davis, CA, 95616, USA

${ }^{7}$ Department of Internal Medicine, University of California Davis, Davis, CA, 95616, USA

${ }^{8}$ Department of Nutrition, University of California Davis, Davis, CA, 95616, USA

${ }^{9}$ Department of Psychology, University of California Davis, Davis, CA, 95616, USA

${ }^{10}$ Department of Obstetrics and Gynecology, School of Medicine, University of California Davis, Davis, CA, 95616, USA

${ }^{11}$ Perinatal Origins of Disparities Center, University of California Davis, Davis, CA, 95616, USA

‡ These authors contributed equally

* Correspondence: jmlasalle@ucdavis.edu 


\begin{abstract}
Maternal obesity during pregnancy is associated with neurodevelopmental disorder (NDD) risk. We utilized integrative multi-omics to examine maternal obesity effects on offspring neurodevelopment in rhesus macaques by comparison to lean controls and two interventions. Differentially methylated regions (DMRs) from longitudinal maternal blood-derived cell-free fetal DNA (cffDNA) significantly overlapped with DMRs from infant brain. The DMRs were enriched for neurodevelopmental functions, methylation-sensitive developmental transcription factor motifs, and human NDD DMRs identified from brain and placenta. Brain and cffDNA methylation levels from a large region overlapping mir-663 correlated with maternal obesity, metabolic and immune markers, and infant behavior. A DUX4 hippocampal co-methylation network correlated with maternal obesity, infant behavior, infant hippocampal lipidomic and metabolomic profiles, and maternal blood measurements of DUX4 cffDNA methylation, cytokines, and metabolites. Ultimately, maternal obesity altered infant brain and behavior, and these differences were detectable in pregnancy through integrative analyses of cffDNA methylation with immune and metabolic biomarkers.
\end{abstract}




\section{Introduction}

In North America, more than half of pregnant women are considered to be overweight or obese. ${ }^{1,2}$ Maternal obesity and related metabolic conditions are associated with a significantly increased risk of offspring with neurodevelopmental disorders (NDD), including autism spectrum disorders (ASD). ${ }^{3-7}$ NDDs are increasing in prevalance, ${ }^{8}$ and ASD is currently diagnosed in 1 in 54 children in the United States of America, where the diagnosis is $\sim 4 x$ more prevalent in males than females. ${ }^{9}$ The elevated risk of an NDD/ASD resulting from maternal obesity is hypothesized to be related to a complex cascade of metabolic and inflammatory events that alter developmental gene regulatory networks. In mice, maternal obesity is associated with sex-specific differences in embryonic brain gene expression, affecting genes related to immunity and inflammation, metabolism, oxidative stress, and development. ${ }^{10}$ Also in mice, maternal high fat diet results in differences in maternal metabolism and inflammation that alter adult offspring brain inflammation and behavior. ${ }^{11,12}$ In Japanese macaques, maternal high fat diet resulted in metabolic and cytokine differences with long-lasting effects on offspring behavior. ${ }^{13}$ In humans, altered metabolites have been observed in the serum of mothers of young children with ASD, including those related to the one-carbon metabolism, critical for the epigenetic modification DNA cytosine methylation. ${ }^{14}$ In mice, a perinatal high-fat diet was found to alter one-carbon metabolism and DNA methylation in the prefrontal cortex of male offspring. ${ }^{15}$ The effect of maternal obesity on one-carbon metabolism in offspring was also seen in baboons fed a high-fat, high-energy diet. ${ }^{16}$ Together, these findings demonstrate that maternal diet alters cytokine and metabolic profiles during pregnancy and suggest that these contribute to altered behavior and brain DNA methylation profiles in offspring from obese mothers. However, challenges remain in understanding the epigenetic mechanisms that explain inter-individual differences following exposure to maternal obesity in humans.

As the maternal-fetal interface, the placenta is the fetal organ that is first affected by the altered metabolites and cytokines resulting from maternal obesity. In humans, pre-pregnancy obesity associated with elevated inflammatory cytokine levels in maternal serum and differential expression of genes related to nutrient transport and immunity in the placenta. ${ }^{17}$ Maternal prepregnancy obesity and trimester-specific gestational weight gain is associated with differential DNA CpG methylation in the placenta. ${ }^{18}$ Maternal obesity is also associated with differential CpG methylation and expression of adiponectin and leptin genes in human placenta. ${ }^{19}$ Adiponectin and leptin are adipokines, which are cytokines secreted by adipose tissue that function as cellular signaling molecules, and their overexpression results in inflammation and altered metabolism. ${ }^{20,21}$ Alterations to inflammatory cytokine levels, which include adipokines, have been associated with ASD, ${ }^{22-24}$ and cytokines play a critical role at the placenta. ${ }^{25,26}$ Functionally, the placental DNA methylome retains profiles of early embryonic development, including neurodevelopment. ${ }^{27-29}$ Previously, we have shown that genome-wide DNA methylation profiles can distinguish human placental samples from newborns later diagnosed with ASD compared to typically developing controls and that DNA methylation profiles are shared between placenta and embryonic brain in a mouse model of a human NDD/ASD relevant environmental exposure. ${ }^{30,31}$ This epigenetic convergence between placenta and brain suggests that placental DNA methylation can inform about individual NDD/ASD risk.

Ideally, epigenetic biomarkers of individual NDD/ASD risk would be obtained during 
pregnancy in order to design behavioral and therapeutic strategies for improved child outcomes. However, direct fetoplacental sampling is invasive and increases the risk for pregnancy loss. For this reason, non-invasive prenatal testing (NIPT) has become an increasingly attractive option for fetal diagnostics. ${ }^{32}$ NIPT is based on assaying the cell-free fetal DNA (cffDNA) that circulates in the blood of pregnant mothers, ${ }^{33,34}$ and originates from the trophoblasts of the placenta. ${ }^{35-37}$ cffDNA is generated by developmental apoptosis, during turnover of the syncytiotrophoblast, and is released into maternal circulation as a membrane bound entity. ${ }^{38,39}$ Furthermore, cffDNA contains a DNA methylation profile representative of its placental origin. ${ }^{40-42}$ cffDNA represents between $12-41 \%$ of cell-free DNA (cfDNA) in the plasma of pregnant women with the percent contribution increasing throughout pregnancy and the remainder of the profile originating from neutrophils, lymphocytes, and the liver. ${ }^{43}$ Taken together, these findings suggest that DNA methylation profiles of placenta, sampled during pregnancy through cffDNA, can be developed to inform about NDD/ASD risk.

\section{Results}

Rhesus macaque maternal obesity models

In order to characterize the multi-factorial molecular cascade that results from maternal obesity, we generated longitudinal cffDNA methylomes from four pregnancy timepoints across all trimesters as well as three infant brain regions (hippocampus, prefrontal cortex, and hypothalamus) at 6 months old (Fig. 1a). We integrated the DNA methylome results obtained from whole genome bisulfite sequencing (WGBS) with two behavioral tests assessing infant recognition memory, immunological markers in maternal blood across pregnancy, maternal blood and infant brain metabolomics, and infant brain lipidomics. The model was based on naturally obese rhesus macaque dams that we previously demonstrated produce offspring with a relevant neurobehavioral profile. ${ }^{44}$ In addition to comparing obese $(n=7)$ to lean matched controls $(n=6)$, we also examined the effects of dietary (caloric restriction, $n=5$ ) and pharmacological (pravastatin, $n=7$ ) obesity interventions, and examined a total of 25 male offspring.

cffDNA methylation profiles are consistent with the placental methylome

First, to assess the quality of the cffDNA methylomes, we performed two analyses to confirm that we could recapitulate previous findings. To confirm that the DNA methylation profile of the cffDNA represents its placental origin more closely than cfDNA in a non-pregnant female, we performed a pilot experiment to compare the DNA methylation profiles of the three sample sources (placental biopsies, cffDNA, and cfDNA) in lean macaques. Principal component analysis (PCA) of the average smoothed methylation levels from regulatory regions and gene bodies revealed that the cffDNA shows a closer relationship to the placenta than cfDNA (Supplementary Fig. 1a). Next, using the primary samples for the study, we leveraged the fact that the pregnancies were all screened to be male fetuses and utilized the ratio of reads from the $Y$ and $X$ chromosomes to show that the fetal fraction of the cffDNA increased throughout the different trimesters of pregnancy (Supplementary Fig. 1b). Taken together, these results demonstrate that 
the cffDNA methylation profiles are consistent with the fetal origin of the placental methylome.

Both cffDNA and brain DMRs map to genes involved in neurodevelopment, cellular adhesion, and cellular signaling

In order to test the hypothesis that maternal obesity and interventions alter DNA methylation patterns in cffDNA and brain, we performed pairwise contrasts of obese vs. control, (caloric) restriction vs. obese, and pravastatin vs. obese, for the cffDNA samples from maternal blood during trimester 1 (GD45), trimester 2 (GD90), early trimester 3 (GD120), and late trimester 3 (GD150) as well as brain region samples from the hippocampus, prefrontal cortex, and hypothalamus of the same infants at 6 months (Supplementary Table 1). Each pairwise DMR comparison generated background regions with similar gene length and $\mathrm{CpG}$ content, and these were used in most downstream enrichment testing to control for genomic context. We then examined the DMRs for each pairwise comparison for potential consistency across time and tissue. A large subset of the DMRs from the pairwise contrasts map to genes that overlap for obese vs. control (Fig. 1b), caloric restriction vs. obese (Fig. 1c), and pravastatin vs. obese (Fig. 1d). The overlaps are not only apparent across different pregnancy timepoints and brain regions for their respective sources but a subset also converge between cffDNA and brain. Next, the genomic coordinates of the DMRs across all pairwise comparisons were merged into separate consensus regions for cffDNA and brain and the same was done for their respective background regions. The overlap between cffDNA and brain consensus DMRs was significant (empirical $p=$ 0.0001 ) in two separate analytical approaches, which included a permutation approach $(n=$ 10,000 ) based on region overlap that placed the DMRs randomly across the entire genome, while maintaining their size, and a random sampling approach $(n=10,000)$ based on nucleotide overlap that utilized background regions with similar genomic context (CpG content and length).

The convergence of DNA methylation alterations associated with maternal obesity was consistent with the gene ontology (GO) analyses of the cffDNA (Fig. 2a) and brain (Fig. 2b) consensus DMRs, relative to their background regions, which were enriched $(q<0.05)$ for terms related to neurodevelopment, cellular adhesion, and cellular signaling. Additionally, the top GO terms from the cffDNA DMRs (anatomical structure morphogenesis, nervous system development, anatomical structure development, plasma membrane bounded cell projection organization, cell adhesion, and movement of cell or subcellular component) and the brain DMRs (nervous system development, cell junction, and cytoskeletal protein binding) also passed a more stringent significance (FWER < 0.1) threshold (Supplementary Table 2), which was based on 100 random sets from samplings of their respective consensus background regions. The GO terms were also consistent with the significant $(q<0.05)$ PANTHER (Protein Analysis THrough Evolutionary Relationships) pathways, which demonstrated a shared effect on integrin signaling, glutamatergic synapses, and angiogenesis in both cffDNA and brain (Fig. 2c-d, Supplementary Table 3). Next, to examine the gene regulatory relevance of the consensus DMRs, they were tested for enrichment within human transcription factor motifs from a methylation-sensitive SELEX (Systematic Evolution of Ligands by EXponential enrichment) experiment. ${ }^{45}$ The top transcription factors for both the cffDNA (Fig. 2e) and brain (Fig. 2f) consensus DMRs, relative to their background regions, were from the hairy and enhancer of split (HES) and activating protein2 (AP-2) families, and overall the top enrichments were related to methylation sensitive 
developmental transcription factors (Supplementary Table 4).

The relevance of the obesity consensus DMRs to previously identified human NDD-associated DMRs was tested by lifting over the consensus DMRs to the human genome (hg38). The liftedover consensus DMRs were tested for enrichment within DMRs from updated analyses of previously published male idiopathic ASD brain, male Dup15q syndrome brain, female Rett syndrome brain, and male Down Syndrome brain and unpublished idiopathic male and female ASD placenta. ${ }^{30,46-48}$ In a permutation approach based on region overlap, the consensus cffDNA and brain DMRs significantly $(q=0.0012)$ overlapped with all human NDD datasets. In a random sampling approach of background regions based on nucleotide overlap, the DMR overlaps showed similar significance $(q<0.05)$, although neither set of consensus DMRs showed a significant enrichment for the female ASD placenta DMRs (Table 1). Notably, the male Down syndrome brain DMRs had a lower enrichment score than the other ASD-related brain DMR datasets in both analytical approaches.

\section{A large block of DNA hypermethylation overlapping mir-663 is shared between cffDNA and brain}

In addition to the DMR analyses, we also performed a separate analysis to detect largerscale blocks of differential methylation in cffDNA and brain associated with maternal obesity and intervention (Supplementary Table 5). The top overall hit in most pairwise contrasts was a large block of differential methylation (chr20:29790471-29824182, width $=33,712$ bp) that was hypermethylated by maternal obesity in both cffDNA and brain. The block was primarily represented by a CpG dense $14.6 \mathrm{~kb}$ region (Fig. 3a). In cffDNA, the maternal obesity group showed the highest methylation level, the intervention groups showed an intermediate level of methylation, and the control group showed the lowest level of methylation. In brain, the maternal obesity group showed the highest level of methylation. The block mapped to a cluster of genes that code for mir-663, ribosomal RNAs (28S, 18S, and 5.8S), 2 novel IncRNAs, a pseudogene, and 3 novel protein coding genes. These results demonstrate the stability across time and tissue type of a maternal obesity DNA methylation difference spanning an entire 33.7 $\mathrm{kb}$ chromosomal locus that presented an intermediate methylation level in the intervention groups during pregnancy. The methylation levels for all cffDNA timepoints and brain regions showed significant positive correlations with maternal obesity (Fig. 3b-c). The longitudinal cffDNA methylation levels of the mir-663 block correlated with inflammatory maternal immune markers and distinct metabolites during their respective pregnancy timepoint (Fig. 3b \& Supplementary Fig. 2). The infant brain region methylation levels of the block showed significant negative correlations with caloric restriction and abstract stimuli recognition memory as well as a significant positive correlation with social stimuli (face) recognition memory (Fig. 3c \& Supplementary Fig. 3). The brain methylation values also correlated with distinct metabolites measured from their respective regions, and the prefrontal cortex showed a negative correlation with C20:3 Homo- $\gamma$-linolenic acid/8,11,14-eicosatrienoic acid concentrations in that brain region.

A DUX4 co-methylation network in infant hippocampus correlates with maternal obesity, behavior, metabolites, lipids, and cffDNA methylation

In order to further investigate gene networks associated with maternal obesity and 
integrate with additional data sets, weighted gene co-methylation network analysis (WGCNA) of WGBS data from infant hippocampus was performed. The signed network demonstrated scalefree topology (Supplementary Fig. 4), leading to the identification of 5 modules of co-methylated regions and their respective hub regions (Table 2). The identified modules were tested for correlations with a suite of traits that included behavioral tests relevant to the hippocampus (abstract stimuli and social stimuli recognition memories) as well as lipidomic and metabolomic measurements from the hippocampus (Fig. 4a \& Supplementary Fig. 5). Notably, the blue module showed a number of significant $(p<0.05)$ negative and positive correlations with all classes of traits measured. The blue module eigengene was negatively correlated with the maternal obesity group, positively correlated with the abstract stimuli recognition memory, and negatively correlated with the social stimuli (face) recognition memory (Fig. 4a-b). The blue module eigengene was also positively correlated with the concentration of multiple polyunsaturated essential fatty acids (PUFAs) in the hippocampus and linoleic acid was the most prominent (Fig. $4 a-b)$. The blue module eigengene was also positively correlated with the concentrations of the metabolites asparagine, citrate, glycerol, and guanosine, and negatively correlated with the concentrations of $\beta$-hydroxybutyric acid, glutathione, and uridine monophosphate (UMP) in the hippocampus (Fig. 4a-b).

The blue module was composed of 104 regions out of 151,892 regions examined (Fig. 4c \& Supplementary Table 6). On average, the regions in the blue module were 1,841 bp and they mapped to 82 unique genes. The hub for the blue module was an intergenic region that mapped to ENSMMUG00000060367, which is a novel gene that is an ortholog for human DUX4 (double homeobox 4) and is termed DUX4 (region) 6. Notably, the blue module was composed of 21 interconnected regions that mapped to DUX4, which spanned 206,145 bp (chr9:427125-633269), were between $-158,396$ bp to 44,716 bp from the DUX4 transcription start site (TSS), and were hypomethylated overall in hippocampi from the offspring of obese mothers. The regions in the DUX4 co-methylated network mapped to genes that were primarily associated with functions related to gene regulation, metabolism, immunity and inflammation, NDDs, oxidative stress, obesity and adipogenesis, Wnt signaling, glutamatergic synapses, endoplasmic reticulum, and reproduction (Table 3 ).

Next, the blue module eigengene was tested for correlations with traits from maternal blood across 4 time points that represented all trimesters of pregnancy, specifically with DUX4 cffDNA methylation levels, cytokine levels, and metabolite levels (Fig. 4d \& Supplementary Fig. 6). These relationships were dynamic across pregnancy, as cffDNA DUX4 methylation levels within region 1 of the module (chr9:631319-633269, width = 1,951 bp) were positively correlated with the blue module eigengene in trimester 1 but negatively correlated with the module in early trimester 3. There were strong negative correlations between the blue module eigengene and levels of several immunological biomarkers of obesity associated inflammation, with the strongest association being MCP-1 (CCL2) levels during trimester 1 , IL-10 during trimester 2, SCD40L during early trimester 3 , and IL-8, TGF- $\alpha$, and High-sensitivity C-reactive Protein (hs-CRP) during early and late trimester 3 . Several metabolites in maternal blood throughout pregnancy showed both positive and negative correlations with the blue module eigengene, including a positive correlation with creatine during trimester 1 and 2 , as well as negative correlations with glutamine and arginine during trimester 1 . The maternal metabolites showing the most prominent correlations with the hippocampal blue module eigengene were generally related to one-carbon 
metabolism (choline, creatine, and glycine) and metabolism of amino acids by the tricarboxylic acid cycle ( $\alpha$-Ketoglutaric Acid, arginine, glutamine, and succinate), which is also known as the citric acid cycle and the Krebs cycle. ${ }^{49,50}$

\section{Discussion}

There are four key findings from this integrative multi-omic analysis of offspring DNA methylation and outcomes resulting from exposure to maternal obesity and intervention in a non-human primate model that are relevant to human NDD/ASD. First, the longitudinal analysis of cffDNA throughout pregnancy demonstrated that cffDNA methylation was consistent with the functions and pathways disrupted in infant brain, particularly for DNA methylation patterns over large genomic blocks at mir-663 and DUX4. Second, the caloric restriction and pravastatin intervention groups displayed intermediate methylation levels in these regions throughout pregnancy. Third, cffDNA methylation levels at DUX4 correlated with the co-methylated DUX4 gene network in 6-month infant hippocampus, which correlated with infant social and abstract recognition memory, infant hippocampal and maternal blood metabolites, and infant hippocampal lipids. Fourth, the maternal obesity DMRs overlapped with DMRs from human ASD/NDD brain and placenta.

To expand on the above summary, the results demonstrate that maternal obesity is associated with the differential methylation of a subset of common genes in both cffDNA and brain. The differentially methylated genes associated with maternal obesity in cffDNA and brain are enriched for functions related to neurodevelopment, cellular adhesion, and cellular signaling. Notably, they converge on pathways known to be affected in NDD/ASD. These include glutamatergic synapses, ${ }^{51}$ angiogenesis, ${ }^{52}$ integrin signaling (which is involved in cellular adhesion), ${ }^{53}$ EGF receptor signaling, and PDGF signaling. Additionally, the brain displayed differences in methylation that were consistent with other neurotransmitters and hormone signaling pathways related to NDDs/ASD, which include thyrotropin (also known as thyroid stimulating hormone; TSH), oxytocin, ${ }^{54,55}$ serotonin, and acetylcholine.

Functionally, the DMRs are consistent with disruptions to the regulation of gene expression, since they are enriched for the motifs of human transcription factors that are methylation sensitive and involved in early development. ${ }^{45}$ The top motif enrichments overall were for the HES and AP-2 families. The HES genes are transcriptional repressors involved in early embryonic development and neurodevelopment that function to regulate the differentiation and proliferation of neural stem cells. ${ }^{56,57}$ The binding of HES transcription factors to their motifs is inhibited by DNA methylation. ${ }^{45}$ The HES genes are effectors of Notch signaling pathway and cross-talk with JAK-STAT signaling, which is activated by cytokines. ${ }^{58}$ Additionally, HES1 is a thyroid response gene in the fetal brain, ${ }^{59}$ which was the top pathway for the fetal brain DMRs associated with maternal obesity and intervention in our study. The AP-2 transcription factors are also involved in early embryonic development, where they stimulate cell-type specific proliferation and repress terminal differentiation. ${ }^{60}$ The top motif from this family belongs to transcription factor gene AP-2 gamma (TFAP2C), which is specifically involved in both placental development and redundantly for retinoic acid induced differentiation of the neural tube, ${ }^{61,62}$ and thus represents a direct connection between the two sample sources.

In addition to discovering a profile of thousands of DMRs of several hundred bp in width, 
in cffDNA and brain, we also identified larger-scale blocks of differential methylation in cffDNA and brain that associated with maternal obesity and intervention. The mir-663 block was 34 kb in size and overlapped several genes, many of which have unknown functions and warrant future functional research. However, mir-663 is associated with obesity and adipocyte differentiation, immunity and inflammation, the mechanism of resveratrol action, and cancer in humans. ${ }^{63-67}$ In our study, hypermethylation of the block in cffDNA correlated with maternal obesity as well as increased maternal inflammatory markers and differential metabolite levels during pregnancy. In infant brain, hypermethylation of the block not only correlated with maternal obesity and infant metabolites, but also with infant behavior and negatively with the caloric restriction intervention. The effect on brain lipids was most pronounced in the prefrontal cortex and through decreased concentrations of C20:3 Homo- $\gamma$-linolenic acid/8,11,14-eicosatrienoic acid, which has anti-inflammatory effects. Through a WGCNA approach, we also identified a key co-methylated network whose hub was a large $200 \mathrm{~kb}$ block of differential methylation that mapped to DUX4. DUX4 is a homeobox transcription factor that is expressed in cleavage stage embryos and testes, and is epigenetically silenced in most other tissues. ${ }^{68}$ Incomplete silencing of DUX4, which is located in the D4Z4 repeat in humans, results in Facioscapulohumeral muscular dystrophy (FSHD) through pathogenic misexpression of DUX4 in skeletal muscle due to DNA hypomethylation of the locus. ${ }^{69}$ This misexpression ultimately leads to an immune deregulation cascade, ${ }^{68}$ and can be repressed by targeted epigenetic editing. ${ }^{70}$ Notably, the DUX4 DNA hypomethylation in FSHD directionally corresponds with the DUX4 co-methylation network we observed in hippocampus, where the maternal obesity with no intervention group was hypomethylated when compared to the control and obesity intervention groups.

The regions in the DUX4 co-methylation network mapped to genes with functions highly related to its significantly correlated phenotypes. The ERBB2 (Erb-B2 Receptor Tyrosine Kinase 2) mapping is consistent with the differences in recognition memory as the gene is known to regulate hippocampal glutamatergic long-term depression and object recognition memory. ${ }^{71}$ Additionally, the glutamatergic synapse is represented in the network by OLFM3 and PRKX, as well as in the top pathways for the consensus cffDNA and brain DMRs. The top pathway from the brain DMRs is also represented in the co-methylation network by SLC16A2, which is a thyroid hormone transporter. Of relevance to the lipidomic profile is a region mapping to FFAR4 (Free Fatty Acid Receptor 4), which is a GPCR (GPR120) for PUFAs that is involved in adipogenesis, metabolism, and inflammation. ${ }^{72,73}$ Levels of linoleic acid (LA, 18:2n-6), an omega-6 PUFA that is a ligand for FFAR4, correlated with the co-methylation network. LA is known to increase neurite outgrowth in the developing brain, ${ }^{74-76}$ and lower levels of LA have also been observed in the serum of children with autism. ${ }^{77}$ The impact of the DUX4 co-methylation network on neurodevelopment is also apparent through the correlation of asparagine, as the network contains the gene ASNS (Asparagine Synthetase). ASNS deficiency is a neurometabolic disorder characterized by severe congenital microcephaly and developmental delay. ${ }^{78}$ The metabolic differences in the brain are also represented by the correlation of the co-methylation network with uridine monophosphate (UMP) and a region mapping to UPP2 (Uridine Phosphorylase 2). Other notable genes in the co-methylation network include: $P C D H 11 X$, which is associated with ASD, ${ }^{79}$ ZFHX3 and ZFHX4, which are homeobox genes that act as transcription factors that regulate myogenic and neuronal differentiation, and ASIP (Agouti Signaling Protein), which is involved in obesity. ${ }^{80}$ 
The DUX4 co-methylation network also showed several significant negative correlations with maternal blood markers during pregnancy that are known to be associated with obesity. Methylation of the DUX4 module was negatively correlated with inflammatory cytokines/adipokines in maternal blood throughout different trimesters of pregnancy, including MCP-1 (CCL2), which is both a monokine and adipokine, ${ }^{81}$ as well as the chemokine IL- 8 and the regulatory cytokine IL-10, which are associated with excess bodyweight. ${ }^{82}$ Additionally, the DUX4 module showed a negative correlation with C-reactive protein (CRP), a marker of inflammation that has been observed to be altered in the serum of mothers with autistic children during pregnancy. ${ }^{83,84}$ Increased levels of MCP-1, IL-8, and CRP have been observed in the blood of obese human mothers during pregnancy. ${ }^{17,85}$ Finally, the differences in maternal metabolites are consistent with previously known impacts on one-carbon metabolism. ${ }^{14-16}$

Taken together, the correlations of the DUX4 co-methylation network in infant hippocampus demonstrate that the lean control and intervention groups had higher levels of methylation than obese pregnancies in the DUX4 co-methylation network, which significantly correlated with differences in behavior related to abstract stimuli and social stimuli recognition memory, higher PUFA concentrations in the brain, and differing levels of metabolites related to neurodevelopment. Furthermore, the higher methylation levels of this module significantly correlated with lower levels of maternal inflammatory cytokines/adipokines and differences in maternal one-carbon metabolism and metabolism of amino acids by the tricarboxylic acid cycle during pregnancy.

Ultimately, the methylation profiles of both the mir-663 block and the DUX4 co-methylation network from the infants of obese mothers without an intervention correlated with decreased recognition memory for abstract stimuli and increased recognition memory for novel social stimuli (faces). The findings demonstrate that, in infant brain, maternal obesity is associated with a DNA methylation profile at gene loci relevant to recognition memory, lipids, and metabolites. These differences in brain multi-omics and behavior can be detected during pregnancy through integrative analyses of cffDNA with immune and metabolic biomarkers. Furthermore, maternal obesity interventions associated with an attenuation of the multi-omic profile in both infant brain and maternal blood. Together, these findings lay the groundwork for retrospective and prospective studies aimed at developing human ASD/NDD diagnostic predictors by utilizing machine learning approaches to select a panel of integrative markers from maternal blood.

\section{Methods}

Non-human primate obesity models

All animals were housed at the California National Primate Research Center (CNPRC) in accordance with the ethics guidelines established and approved by the Institutional Animal Use and Care Administrative Advisory Committee at the University of California-Davis.

Adult pregnant female rhesus macaques (Macaca mulatta) with male fetuses were selected for this study. Sex of the fetus was determined with a cell-free fetal DNA Y chromosome gene analysis of maternal blood and was performed early in the first trimester by the CNPRC Primate Assay Core. All dams ranged in age from 7 to 12 years and were selected for lean and obese groups based on their Body Condition Score (BCS). ${ }^{86}$ Obese females had a BCS of at least 
3.5 (range 1 - 5) which correlates with $32 \%$ body fat, and lean animals had a BCS of $2-2.5$. Animals had maintained a consistent BCS for at least one year prior to selection for the study and pre-study physicals confirmed that none of the selected females were diabetic. All animals were maintained with standard indoor housing conditions at CNPRC and fed nine "biscuits" of commercial chow (High Protein Primate Diet Jumbo; LabDiet; 5047) twice daily while pregnant, received biweekly fresh produce, daily forage mixture, and ad libitum water. The caloric restriction group had the amount of chow restricted to prevent weight gain during pregnancy and the Pravastatin group was given $1 \mathrm{mg} / \mathrm{kg}$ of body weight. However, all dams, regardless of group, were provided twelve biscuits twice daily during nursing of 4 months or older infants. Dams were relocated to a single housing room around gestational day 70. Approximately two weeks later they were paired with a compatible cage mate during daytime hours and were separated prior to feeding times. Dams were allowed to deliver naturally ( 165 day gestation length) and mother-infant pairs were raised indoors until offspring were 6 months old. However, five pregnant dams required Cesarian deliveries for post-date pregnancies ( 175 gestation days) or as recommended by veterinarians for health reasons, two in the obese group, one in the pravastatin group and two in the lean control group. In those scenarios, infants were successfully reared to 6 months of age by foster dams using established CNPRC protocols. ${ }^{87}$ Mother-infant dyads were housed indoors with another compatible mother-infant dyad during daytime hours if possible; however, this was not possible for 1 lean control dyad, 3 obese dyads, and 1 pravastatin dyad. Infant brain samples (hippocampus, hypothalamus, and prefrontal cortex) were collected on postnatal day 180 after infants were anesthetized with ketamine and euthanized with $120 \mathrm{mg} / \mathrm{kg}$ pentobarbital. Upon collection, samples were immediately frozen and stored at $-80^{\circ} \mathrm{C}$.

\section{Abstract stimuli recognition memory}

Infants were tested at 200 days gestational age (days from conception; 1 month old) to avoid differential maturity due to variation in gestation length (range: 152-176 days). The mean postnatal age was 36 days (range: 22-49 days). Infants were separated from mothers, wrapped in a towel and carried to the testing station. The testing apparatus consisted of a small booth with side panels to shield from outside distractions in a darkened room in which the stimuli, 9 $\mathrm{cm}^{2}$, were mounted on the left and right of a center viewing hole. One tester held the infant 36 $\mathrm{cm}$ away from the stimuli, changed the stimuli and covered the infant's head between trials. A second tester sat behind the apparatus and viewed the infant's head through a video camera to record fixation times to the left and right. Two identical stimuli were placed on the right and left until the infant accumulated a familiarization time of $20 \mathrm{sec}$. One stimulus, randomly determined, was then removed and replaced with the novel stimulus. The frequency and duration of looking was recorded for $10 \mathrm{sec}$, then the stimuli were exchanged between sides for another $10 \mathrm{sec}$ test period. The infant was presented with a series of four black and white visual stimuli pairs (abstract illustrations of varying complexity) used in the Fagan Test of Infant Intelligence for human infants (Infantest Corp). Videos were scored for right/left looking using The Observer coding software (Noldus) by a coder blind to stimulus location and animal treatment group. The evaluation relies on the propensity of monkey as well as human infants to look longer at novel than familiar visual stimuli. The outcome measure used in this study was the average across the 
four problems of the number of looks at the novel stimulus divided by the number of looks at the novel and familiar stimuli during test trials. ${ }^{88}$ All infants in the lean control group had a ratio $>0.50$ indicating novelty preference.

\section{Social stimuli recognition memory}

Animals were tested at a mean age of 105.3 days (range: $96-124)$. They were separated from their dams and relocated to individual housing indoors in a standard size housing cage $(0.58 \mathrm{~m} \times 0.66 \mathrm{~m} \times 0.81 \mathrm{~m}$, Lab Products). Approximately 2.5 hours after the separation/relocation, they were given a visual paired comparisons task. Each animal was hand-carried to a test cage measuring $0.387 \mathrm{~m} \times 0.413 \mathrm{~m} \times 0.464 \mathrm{~m}$ that was positioned $0.686 \mathrm{~m}$ from a $0.813 \mathrm{~m}$ monitor (Panasonic, KV 32540), was given 30-sec to habituate, and was then presented with seven problems from a pre-recorded video. Each problem included three trials: a familiarization trial and two recognition trials. After a 5-sec blank screen, a $20-\mathrm{sec}$ familiarization trial began, in which two identical pictures were presented, each measuring $19.7 \times 22.9 \mathrm{~cm}$, separated by $25.4 \mathrm{~cm}$ of white space onscreen. After another 5-sec delay, an 8-sec recognition trial occurred, in which the now-familiar stimulus was presented simultaneously with a novel stimulus (side determined randomly). Following another 5-sec delay, the same two stimuli were presented again for 8-sec, with positions reversed. Seven such problems were presented. All stimuli were pictures of unfamiliar juvenile and adult monkeys of both sexes. ${ }^{89} \mathrm{~A}$ tone of $1000 \mathrm{~Hz}$ was presented 250 milliseconds prior to trials in order to orient the animal. A low-light camera (KT\&C, KTLCMB5010EX), attached to the display monitor and situated midway between the two projected images, was used to record the subjects' looking responses. Looking behavior during the familiarization and recognition trials was scored by a trained observer who was blind to the animals' treatment groups. For each problem, the proportion of looking time directed at the novel stimulus was computed: duration of viewing the novel stimulus on the two recognition trials divided by the duration of viewing both the novel and familiar stimuli in the recognition trials. The principal outcome measure was a mean of this proportion across the seven problems. Chance responding was indicated by a mean of 0.50 , with lower values suggesting a preference for the familiar stimuli and higher values indicating preference for the novel stimulus. Upon completion of testing, the subject was returned to its holding cage, and the test area was cleaned and prepared for the next subject.

\section{Lipidomics}

The lipid extraction protocol was performed as previously described. ${ }^{90}$ Briefly, $30 \mathrm{mg}$ of homogenized half brain samples were used. Upon obtaining Folch bottom layers, lipids were evaporated under nitrogen, reconstituted into $1.5 \mathrm{~mL}$ chloroform (Fisher Scientific; Cat \#C607-4): isopropyl alcohol (Fischer Scientific, Cat \#464-1) (v/v; 2/1). Approximately $0.5 \mathrm{~mL}$ of extract, with $0.0125 \mathrm{mg} 5 \alpha$-Cholestane (Sigma-Aldrich, Cat \#C8003-100mg), and 0.1mg C17:0 PC (1,2diheptadecanoyl-sn-glycero-3-phosphocholine (Avanti Polar Lipids; 850355C) was dried under nitrogen for fatty acid and cholesterol analysis. Upon drying, $400 \mu \mathrm{L}$ of toluene (Fisher Scientific; T2914) was added, followed by $3 \mathrm{~mL}$ of methanol (Fisher Scientific; Cat \#A454-4), and $600 \mu \mathrm{L}$ of $3 \% \mathrm{HCl}$ (Sigma-Aldrich; 320331) in methanol. The transesterification reaction to generate fatty 
acid methyl esters (FAMEs) was adapted from previous research. ${ }^{91}$ Samples were vortexed and heated at $90^{\circ} \mathrm{C}$ for $60 \mathrm{~min}$. After cooling the samples at room temperature for $4-5 \mathrm{~min}, 1 \mathrm{~mL}$ of hexane (Fisher Scientific; H303-4) followed by $1 \mathrm{~mL}$ of deionized water was added to each sample. Samples were vortexed and the phases were allowed to separate for $15 \mathrm{~min}$. Then, $900 \mu \mathrm{L}$ from the hexane top layer containing FAMEs was transferred to microfuge tubes containing $450 \mu \mathrm{L}$ of deionized water. The tubes were vortexed and centrifuged at $15,871 \mathrm{~g}$ for $2 \mathrm{~min}$. The top hexane layer was evaporated under nitrogen and then reconstituted in $100 \mu \mathrm{L}$ hexane for GC-FID (Gas Chromatography with Flame-Ionization Detection) analysis.

A simultaneous FAME and cholesterol GC-FID method was developed by optimizing two previous methods. ${ }^{92,93}$ Samples were analyzed on a Perkin Elmer Clarus 500 GC-FID system (Perkin Elmer) equipped with a DB-FFAP polyethylene glycol fused capillary column (30 m × 0.25 $\mathrm{mm}$ inner diameter, $0.25 \mu \mathrm{m}$ film thickness; Agilent Technologies; 1223232). The injector and detector temperatures were $285^{\circ} \mathrm{C}$ and $300^{\circ} \mathrm{C}$, respectively. The initial oven temperature was $80^{\circ} \mathrm{C}$. It was held at $80^{\circ} \mathrm{C}$ for $2 \mathrm{~min}$, increased by $10^{\circ} \mathrm{C} / \mathrm{min}$ to $185^{\circ} \mathrm{C}$, raised to $249^{\circ} \mathrm{C}$ at $6^{\circ} \mathrm{C} / \mathrm{min}$ and lastly held at $240^{\circ} \mathrm{C}$ for $44 \mathrm{~min}$. The total run time was $65 \mathrm{~min}$. Helium was used as the carrier gas with a maintained flow rate of $1.3 \mathrm{~mL} / \mathrm{min}$. The injection volume was $1 \mu \mathrm{L}$ per sample. The split ratio was 10:1. A custom-made mix of 29 FAME standards was used to identify each fatty acid based on retention time. Fatty acid concentrations were determined by comparing GC peak areas to the internal standard area. Cholesterol concentration was calculated based on a fivepoint standard curve of cholesterol and $5 \alpha$-Cholestane as surrogate. The linear dynamic range of the calibration curve for cholesterol was $0.125 \mathrm{mg} / \mathrm{mL}$ to $2 \mathrm{mg} / \mathrm{mL}$.

\section{Metabolomics}

Fasting blood was collected in lavender top (EDTA) tubes from mothers once during the 1st and 2nd trimesters and twice during the 3rd trimester after anesthetization with 5-30 mg/kg ketamine or 5-8 $\mathrm{mg} / \mathrm{kg}$ telazol. Plasma samples were filtered using Amicon Ultra Centrifugal Filters ( $3 \mathrm{k}$ molecular weight cutoff, Millipore) to remove proteins and lipids. Metabolites were extracted from each of the brain tissues as previously described. ${ }^{90}$ To $207 \mu \mathrm{L}$ of either plasma filtrate or brain tissue extract, $23 \mu \mathrm{L}$ of internal standard containing DSS-d6 was added and samples were placed in $3 \mathrm{~mm}$ Bruker nuclear magnetic resonance (NMR) tubes. Proton NMR spectra were acquired on each sample at $25^{\circ} \mathrm{C}$ using the noesypr1d pulse sequence on a Bruker Avance $600 \mathrm{MHz}$ NMR spectrometer (Bruker, Billerica, MA, USA) and analyzed using Chenomx NMRSuite (version 8.1, Chenomx Inc) as previously described. ${ }^{90}$

\section{Immunology}

A longitudinal analysis on the maternal cytokine/chemokine profile that included 22 analytes (GM-CSF, IFN- $\gamma$, IL-1b, IL-ra, IL-2, IL-4, IL-5, IL-6, IL-8, IL-10, IL-12/23(p40), IL-13, IL-15, IL17a, IL-18, MCP-1, MIP-1b, MIP-1a, sCD40L, TGF $\alpha$, TNF $\alpha$, and VEGF) was measured in plasma using a non-human primate multiplexing bead immunoassay (Millipore-Sigma, Burlington, MA) according to the manufacturer's protocol. The plates were read on a Bio-Plex 200 system (BioRad Laboratories, Hercules, CA, USA) and analyzed using Bio-Plex Manager software (Bio-Rad 
Laboratories). A five-parameter curve was used to calculate final concentrations (pg/ml). Reference samples were run on each plate for assay consistency.

\section{DNA extraction and WGBS library preparation}

The cffDNA was extracted from serum using a Maxwell RSC cffDNA Plasma Kit (Promega; AS1480) by the Primate Assay Laboratory Core at the California National Primate Research Center. The brain DNA was isolated from tissue stored in DNA/RNA shield (Zymo Research; R1100-250) using the Quick-DNA Miniprep Plus kit workflow on a Tecan instrument by Zymo Research. Brain DNA was fragmented using a E220 focused-ultrasonicator (Covaris; 500239). DNA was bisulfite converted using the EZ DNA Methylation-Lightning Kit (Zymo Research; D5031). WGBS library preparation was performed via the Accel-NGS Methyl-Seq DNA Library Kit (Swift Biosciences; 30096) with the Methyl-Seq Combinatorial Dual Indexing Kit (Swift Biosciences; 38096) according to the manufacturer's instructions. The primary cffDNA libraries were prepared by Swift Biosciences and the brain libraries were prepared by the UC Davis Genome Center. The primary cffDNA and brain library pools were sequenced by the UCSF Center for Advanced Technology (CAT) core facility on the Illumina NovaSeq 6000 S4 for 150 bp paired end reads. The pilot cffDNA library pool utilized the Methyl-Seq Set A Indexing Kit (Swift Biosciences; 36024) and was sequenced by the DNA Technologies and Expression Analysis Cores at the UC Davis Genome Center on an Illumina HiSeq 4000 for 90 bp single reads.

\section{Bioinformatic analyses}

The CpG_Me alignment pipeline (https://github.com/ben-laufer/CpG Me \& doi: 10.5281/zenodo.5030083), which is based on Trim Galore, FastQ Screen, Bismark, Picard, and MultiQC, was used to trim adapters and methylation bias, screen for contaminating genomes, align to the reference genome (rheMac10), remove duplicates, calculate coverage and insert size metrics, extract CpG methylation values, generate genome-wide cytosine reports (CpG count matrices), and examine quality control metrics. ${ }^{94-100}$ cffDNA samples were examined for their ratio of chrY/chrX reads (https://github.com/hyeyeon-hwang/SexChecker).

DMR calling and most downstream analyses and visualizations were performed via DMRichR (https://github.com/ben-laufer/DMRichR \& doi: 10.5281/zenodo.5030057), which utilizes the dmrseq and bsseq algorithms. ${ }^{100-102} \mathrm{~A}$ methylation difference of $10 \%$ was used for the cffDNA DMR analyses and 5\% was used for the brain DMR analyses, as previously described for placenta and brain. ${ }^{30,48}$ Background regions with similar genomic context to the DMRs (gene length and CpG content) were obtained from the first step of dmrseq and utilized in downstream enrichment testing. ChIPseeker (was used to obtain gene region annotations and gene symbol mappings through ensembldb. ${ }^{103,104}$ ComplexUpset was used to create UpSet plots of gene overlaps. ${ }^{105-107}$ GOfuncR was used for genomic coordinate based gene ontology (GO) analyses, where DMRs were mapped to genes if they were between $5 \mathrm{~Kb}$ upstream to $1 \mathrm{~Kb}$ downstream of the gene body, and 100 random samplings from the background regions with gene length correction was utilized for the enrichment testing. ${ }^{108,109}$ The DMRs were mapped to genes if they were within $5 \mathrm{~kb}$ upstream or $1 \mathrm{~kb}$ downstream of the gene body. Redundant GO terms were then removed based on semantic similarity using rrvgo. ${ }^{110}$ enrichR was used for gene symbol 
based PANTHER pathway enrichment testing. ${ }^{111-114}$ regioneR was utilized to perform permutation based genomic coordinate enrichment testing through a randomized region strategy with 10,000 permutations. ${ }^{115}$ GAT was used to perform random sampling based genomic coordinate enrichment testing through 10,000 samplings of background regions. ${ }^{116}$ Analysis of Motif Enrichment (AME) in the MEME Suite was utilized to perform transcription factor motif testing relative to background regions using the Human Methylcytosine database with rheMac10 sequences through the memes package. ${ }^{45,117-119}$ The WGCNA package was used to a construct a signed co-methylation network through the biweight mid-correlation (bicor) method. ${ }^{120,121}$

\section{Data Availability}

The raw and processed WGBS data has been deposited in GEO (Accession \#: Available upon publication). All original code has been deposited on GitHub (https://github.com/benlaufer/cffDNA-and-Brain-Manuscript).

\section{Author Contributions}

CKW, CAV, JML, MSG, JPC, CMS, AYT, JAV, and MDB conceptualized the study. CKW and CAV obtained funding for the study. CAV supervised all prenatal animal work and performed infant tissue sampling, and CKW supervised and performed maternal gestation sampling. BIL prepared the pilot cffDNA libraries. BIL and HH performed the DNA methylation analyses under the supervision of JML. YH performed the metabolomic analyses under the supervision of CMS. $\mathrm{ZZ}$ performed the lipidomic analyses under the supervision of AYT. LAD preformed the social stimuli behavior work under the supervision of JPC. CEH performed the abstract stimuli behavior work under the supervision of MDB and MSG. LH performed the immunology analyses under the supervision of JAV. BIL wrote the manuscript with intellectual contributions from JML. All authors wrote their respective methods section and reviewed and approved the final manuscript.

\section{Competing Interests}

The authors declare no competing interests.

\section{Acknowledgements}

This work was supported by a National Institutes of Health (NIH) grant from the Eunice Kennedy Shriver National Institute of Child Health and Human Development [5R01HD084203-04] to CKW and CAV, a NIH grant [R24OD010962] to JPC, a Canadian Institutes of Health Research (CIHR) postdoctoral fellowship [MFE-146824] and a CIHR Banting postdoctoral fellowship [BPF162684] to BIL, the UC Davis Intellectual and Developmental Disabilities Research Center (IDDRC) [P50HD103526], and the California National Primate Research Center (CNPRC) [P51-OD011107]. The cffDNA research was funded by seed grants from the UC Davis IDDRC and Genome Center awarded to CKW and BIL. The brain DNA library preparation was carried out by the DNA Technologies and Expression Analysis Cores at the UC Davis Genome Center and was supported by a NIH Shared Instrumentation Grant [1S100D010786-01]. The authors would like to thank 
bioRxiv preprint doi: https://doi.org/10.1101/2021.08.27.457952; this version posted August 28, 2021. The copyright holder for this preprint (which was not certified by peer review) is the author/funder. All rights reserved. No reuse allowed without permission.

Jasmin Zarrabi and Dana Hill from the California National Primate Research Center for coordinating the project. The authors would also like to thank Dr. Brad Friedman from Genentech for invaluable discussions about the bioinformatic approaches presented in this manuscript. 
Table 1: Human NDD-associated DMR enrichments for the consensus cffDNA DMRs and consensus brain DMRs.

\begin{tabular}{|l|l|l|l|l|l|l|}
\hline \multicolumn{4}{|c|}{ Dataset } & \multicolumn{2}{c|}{ cffDNA DMRs } & \multicolumn{2}{c|}{ Brain DMRs } \\
\hline NDD & Sex & Tissue & Fold & q-value & Fold & q-value \\
\hline ASD & Female & Placenta & 1.05 & 0.3 & 1.08 & 0.1 \\
\hline ASD & Male & Placenta & 1.25 & 0.04 & 1.26 & 0.03 \\
\hline Down Syndrome & Male & Brain & 1.12 & 0.008 & 1.37 & 0.0002 \\
\hline ASD & Male & Brain & 1.36 & 0.0002 & 1.51 & 0.0002 \\
\hline Rett Syndrome & Female & Brain & 1.53 & 0.0002 & 1.69 & 0.0002 \\
\hline Dup15q Syndrome & Male & Brain & 1.28 & 0.0002 & 1.74 & 0.0002 \\
\hline
\end{tabular}

Table 2: WGCNA module hub genes.

\begin{tabular}{|c|c|c|l|l|}
\hline Module & Coordinates & Width & Annotation & \multicolumn{1}{|c|}{ Gene Mapping } \\
\hline Green & chr18:45038579-45038736 & 158 & Intron & $\begin{array}{l}\text { CELF4 } \\
\text { CUGBP Elav-like Family Member 4 } \\
\text { ENSMMUG00000006866 }\end{array}$ \\
\hline Yellow & chr4:139959607-139965208 & 5602 & 3' UTR & $\begin{array}{l}\text { MAMU-A3 } \\
\text { Major Histocompatibility Complex, Class I, A } \\
\text { ENSMMUG00000056914 }\end{array}$ \\
\hline Blue & chr9:443230-472517 & 29288 & Intergenic & $\begin{array}{l}\text { DUX4 } \\
\text { Double Homeobox 4 } \\
\text { ENSMMUG00000060367 }\end{array}$ \\
\hline Brown & chr10:26959768-26960135 & 368 & Intergenic & ENSMMUG00000051997 \\
\hline Turquoise & chrY:8116120-8122200 & 6081 & Intergenic & $\begin{array}{l}\text { LOC106995433 } \\
\text { Heat Shock Transcription Factor, Y-linked-like } \\
\text { ENSMMUG00000049379 }\end{array}$ \\
\hline
\end{tabular}


Table 3: Manually curated blue module co-methylated network gene mapping categories.

\begin{tabular}{|l|l|}
\hline Category & Genes \\
\hline Gene Regulation & AGO1, ARID5B, BRD7, DUX4, ETV1, EXOSC9, JDP2, HLCS, \\
& KLF4, KLF12, NFE2L3, PAXBP1, PRMT9, SFSWAP, WDR82, \\
& ZC3H12C, ZFHX3, ZFHX4, ZNF362, ZNF721 \\
\hline Metabolism & ASNS, DGKH, DPY19L2, EDEM3, EXOSC9, FFAR4, GMDS, \\
& HLCS, ITIH6, KLF4, KLK13, LYVE1, NUDT7, PHEX, PRMT9, \\
& SENP1, UPP2 \\
\hline Immunity and Inflammation & ARID5B, ACVR2A, CD99, CST7, DOCK11, EXOSC9, FFAR4, \\
& IFTAP, IRAG2, LRRC32, LYVE1, NCF2, PRKX, TNFRSF11A, \\
& ZC3H12C \\
\hline Neurodevelopmental Disorders & ASNS, CHL1, EFR3A, ERBB2, EXOC6B, GPC5, LRRC32, \\
& PCDH11X, POMK, PTPRG, SLC16A12, TNIK, VWA3B, WDR82 \\
\hline Oxidative Stress & FAM12OA, NCF2, NFE2L3, NUDT7, PXDNL, TXNL1 \\
\hline Obesity and Adipogenesis & ACVR2A, ARID5B, ASIP, FFAR4, KLF4, KSR2 \\
\hline Wnt Signaling & BRD7, JDP2, PCDH11X, SHISA7, TNIK \\
\hline Glutamatergic Synapses & ERBB2, OLFM3, PRKX \\
\hline Endoplasmic Reticulum & EDEM3, IRAG2, NFE2L3, UFL1 \\
\hline Reproduction & DPY19L2, MAGEB1, MROH5 \\
\hline
\end{tabular}


Figure Legends:

Fig. 1: cffDNA and brain DMR overlaps. A) Experimental design and timeline (created with BioRender.com). UpSet plots of the overlaps of gene mappings from pairwise DMR comparisons of B) obese vs. control, C) caloric restriction vs. obese, and D) pravastatin vs. obese for cffDNA and brain.

Fig. 2: Functional enrichments for the consensus cffDNA and brain DMRs. Top slimmed gene ontology (GO) enrichment testing results for the A) cffDNA and B) brain consensus DMRs. Top PANTHER pathway enrichments for the C) cffDNA and D) brain consensus DMRs. Top human methylation sensitive transcription factor motif enrichment testing results for the E) cffDNA and F) brain consensus DMRs. The motif names indicate whether the transcription factor was fulllength ("-FL") or an extended DNA-binding domain ("-eDBD"), if the CpGs were methylated ("methyl"); and the number (starting with "-1") distinguishes between multiple motifs.

Fig. 3: A large block of obesity associated DMR hypermethylation in cffDNA and brain. A) Plot of methylation levels in the main block region. The dots in the scatter plot are individual DNA methylation level estimates for a CpG site and their size reflects the level of coverage from the sequencing. Percent DNA methylation is presented on the $y$-axis and the $x$-axis is the genomic coordinate of each $\mathrm{CpG}$, where the ticks show the location of the methylation loci. The bottom right track contains CpG annotations and gene mappings and for the block. B) Correlation heatmap of the relationship between longitudinal cffDNA methylation levels in the block and maternal blood immune markers and metabolites measured during the same trimester. The heatmap colors are representative of the correlation between the methylation values and the trait of interest. The correlation values are reported above their $p$-values in parentheses. The obesity group refers to maternal obesity with no intervention. C) Correlation heatmap of the relationship between infant brain region DNA methylation levels in the block and infant hippocampal lipids and metabolites measured from the same brain region. The plots and statistical testing are based on the region of highest CpG density (chr20:29807471-29822071, width $=14,601 \mathrm{bp}$ ), which represents the primary signal of the entire block (chr20:2979047129824182, width $=33,712$ bp).

Fig. 4: Weighted gene co-methylation network (WGCNA) of infant hippocampus. A) Moduletrait correlations within the hippocampus. The heatmap colors are representative of the correlation between the module eigengenes and the trait of interest. The obesity group refers to maternal obesity with no intervention. The correlation values are reported above their $p$-values in parentheses and these values also apply to the expanded plots in part B of the figure. B) Bar plot of the mean eigengene values for the blue module in each maternal group, and scatter plots with a line of best fit for the eigengene values and abstract stimuli recognition memory score ratios, social stimuli recognition memory score ratios, linoleic acid concentrations, asparagine concentrations, and citrate concentrations. C) The blue module maternal obesity co-methylation network. Regions were mapped to their nearest gene and novel genes were labelled with mammalian ortholog symbols, if available. Genes represented by more than one region were appended with a unique number identifier. Edges were included in the network if they passed an 
adjacency threshold and thus not all genes in the module are represented in the visualization. D) Hippocampal blue module-trait correlations with maternal blood measurements of DUX4 cffDNA methylation levels, immunological markers, and metabolites across all trimesters of pregnancy.

Supplementary Fig. 1: cffDNA technical confirmation experiments from the pilot and main experiments. A) Principal component analysis (PCA) of smoothed methylation levels from gene regulatory and gene body regions ( $+5 \mathrm{~kb}$ to $-1 \mathrm{~kb}$ from the gene body) comparing cffDNA, cellfree DNA (cfDNA) from non-pregnant mothers, and placental biopsies from different pregnancy time points in the pilot experiment. The outermost color around each dot represents the individual animal. B) Line plot of the ratio of $\mathrm{Y}$ chromosome reads over $\mathrm{X}$ chromosome reads for the cffDNA methylomes across all pregnancy timepoints for the different groups from the main experiment.

Supplementary Fig. 2: Expanded heatmap of the correlation between longitudinal cffDNA methylation levels in the block and maternal blood immune markers and metabolites measured during the same trimester.

Supplementary Fig. 3: Expanded heatmap of the correlation between infant brain region DNA methylation levels in the block and infant hippocampal lipids and metabolites measured from the same brain region.

Supplementary Fig. 4: Analysis of scale-free topology used to determine the soft-thresholding power for infant hippocampal DNA methylation network construction.

Supplementary Fig. 5: Expanded infant hippocampus module-trait relationship heatmap.

Supplementary Fig. 6: Expanded hippocampal blue module-trait correlations with maternal blood measurements across all trimesters of pregnancy.

Supplementary Table 1: Annotated cffDNA and brain DMRs (empirical $p<0.05)$ from all pairwise obesity comparisons.

Supplementary Table 2: GO enrichments for the consensus cffDNA DMRs and consensus brain DMRs.

Supplementary Table 3: PANTHER pathway enrichments for the consensus cffDNA DMRs and consensus brain DMRs.

Supplementary Table 4: Human methylation sensitive transcription factor motif enrichments within the consensus cffDNA DMRs and consensus brain DMRs.

Supplementary Table 5: Annotated cffDNA and brain blocks (empirical $p<0.05$ ) and background blocks from all pairwise obesity comparisons. 
Supplementary Table 6: Annotated regions from the infant hippocampus blue module maternal obesity network.

\section{References}

1. Dzakpasu, S. et al. Contribution of prepregnancy body mass index and gestational weight gain to adverse neonatal outcomes: population attributable fractions for Canada. BMC Pregnancy Childbirth 15, 21 (2015).

2. Branum, A. M., Kirmeyer, S. E. \& Gregory, E. C. W. Prepregnancy body mass index by maternal characteristics and state: Data from the birth certificate, 2014. Natl. Vital Stat. Reports 65, 1-11 (2016).

3. Krakowiak, P. et al. Maternal Metabolic Conditions and Risk for Autism and Other Neurodevelopmental Disorders. Pediatrics 129, e1121-e1128 (2012).

4. Li, M. et al. The Association of Maternal Obesity and Diabetes With Autism and Other Developmental Disabilities. Pediatrics 137, e20152206-e20152206 (2016).

5. Kong, L., Norstedt, G., Schalling, M., Gissler, M. \& Lavebratt, C. The risk of offspring psychiatric disorders in the setting of maternal obesity and diabetes. Pediatrics 142, (2018).

6. Girchenko, P. et al. Maternal early pregnancy obesity and related pregnancy and prepregnancy disorders: associations with child developmental milestones in the prospective PREDO Study. Int. J. Obes. 42, 995-1007 (2018).

7. Matias, S. L. et al. Maternal prepregnancy weight and gestational weight gain in association with autism and developmental disorders in offspring. Obesity (2021). doi:10.1002/oby.23228

8. Zablotsky, B. et al. Prevalence and trends of developmental disabilities among children in the United States: 2009-2017. Pediatrics 144, (2019).

9. Maenner, M. J. et al. Prevalence of autism spectrum disorder among children aged 8 Years-Autism and developmental disabilities monitoring network, 11 Sites, United States, 2016. MMWR Surveill. Summ. 69, 1-12 (2020).

10. Edlow, A. G. et al. Males are from Mars, and females are from Venus: sex-specific fetal brain gene expression signatures in a mouse model of maternal diet-induced obesity. Am. J. Obstet. Gynecol. 214, 623.e1-623.e10 (2016).

11. Bilbo, S. D. \& Tsang, V. Enduring consequences of maternal obesity for brain inflammation and behavior of offspring. FASEB J. 24, 2104-2115 (2010).

12. Fernandes, D. J. et al. Exposure to maternal high-fat diet induces extensive changes in the brain of adult offspring. Transl. Psychiatry 11, 149 (2021).

13. Thompson, J. R. et al. Maternal diet, metabolic state, and inflammatory response exert unique and long-lasting influences on offspring behavior in non-human primates. Front. Endocrinol. (Lausanne). 9, 161 (2018).

14. Hollowood-Jones, K. et al. Altered metabolism of mothers of young children with Autism Spectrum Disorder: a case control study. BMC Pediatr. 20, 557 (2020).

15. McKee, S. E., Zhang, S., Chen, L., Rabinowitz, J. D. \& Reyes, T. M. Perinatal high fat diet and early life methyl donor supplementation alter one carbon metabolism and DNA methylation in the brain. J. Neurochem. 145, 362-373 (2018). 
16. Nathanielsz, P. W. et al. Maternal obesity disrupts the methionine cycle in baboon pregnancy. Physiol. Rep. 3, (2015).

17. Sureshchandra, S. et al. Inflammatory Determinants of Pregravid Obesity in Placenta and Peripheral Blood . Frontiers in Physiology 9, 1089 (2018).

18. Shrestha, D., Ouidir, M., Workalemahu, T., Zeng, X. \& Tekola-Ayele, F. Placental DNA methylation changes associated with maternal prepregnancy BMI and gestational weight gain. Int. J. Obes. 44, 1406-1416 (2020).

19. Nogues, P. et al. Maternal obesity influences expression and DNA methylation of the adiponectin and leptin systems in human third-trimester placenta. Clin. Epigenetics 11, 1-18 (2019).

20. Lehr, S., Hartwig, S. \& Sell, H. Adipokines: a treasure trove for the discovery of biomarkers for metabolic disorders. Proteomics. Clin. Appl. 6, 91-101 (2012).

21. Unamuno, X. et al. Adipokine dysregulation and adipose tissue inflammation in human obesity. Eur. J. Clin. Invest. 48, e12997 (2018).

22. Goines, P. E. et al. Increased midgestational IFN- $\gamma$, IL-4 and IL-5 in women bearing a child with autism: A case-control study. Mol. Autism 2, 1-11 (2011).

23. Zerbo, O. et al. Neonatal cytokines and chemokines and risk of Autism Spectrum Disorder: The Early Markers for Autism (EMA) study: A case-control study. J. Neuroinflammation 11, (2014).

24. Traglia, M. et al. Cross-genetic determination of maternal and neonatal immune mediators during pregnancy. Genome Med. 10, (2018).

25. Du, M.-R., Wang, S.-C. \& Li, D.-J. The integrative roles of chemokines at the maternalfetal interface in early pregnancy. Cell. Mol. Immunol. 11, 438-448 (2014).

26. PrabhuDas, M. et al. Immune mechanisms at the maternal-fetal interface: Perspectives and challenges. Nature Immunology 16, 328-334 (2015).

27. Schroeder, D. I., Lott, P., Korf, I. \& LaSalle, J. M. Large-scale methylation domains mark a functional subset of neuronally expressed genes. Genome Res. 21, 1583-91 (2011).

28. Schroeder, D. I. \& LaSalle, J. M. How has the study of the human placenta aided our understanding of partially methylated genes? Epigenomics 5, 645-654 (2013).

29. Schroeder, D. I. et al. The human placenta methylome. Proc. Natl. Acad. Sci. U. S. A. 110, 6037-42 (2013).

30. Zhu, Y. et al. Placental DNA methylation levels at CYP2E1 and IRS2 are associated with child outcome in a prospective autism study. Hum. Mol. Genet. 28, 2659-2674 (2019).

31. Laufer, B. I. et al. Genome-Wide DNA Methylation Profiles of Neurodevelopmental Disorder Genes in Mouse Placenta and Fetal Brain Following Prenatal Exposure to Polychlorinated Biphenyls. bioRxiv (2021). doi:10.1101/2021.05.27.446011

32. Chiu, R. W. K. \& Lo, Y. M. D. Cell-free fetal DNA coming in all sizes and shapes. Prenat. Diagn. (2021). doi:10.1002/pd.5952

33. Lo, Y. M. D. et al. Prenatal Sex Determination By DNA Amplification From Maternal Peripheral Blood. Lancet 334, 1363-1365 (1989).

34. Lo, Y. M. D. et al. Presence of fetal DNA in maternal plasma and serum. Lancet 350, 485487 (1997).

35. Masuzaki, H. et al. Detection of cell free placental DNA in maternal plasma: direct evidence from three cases of confined placental mosaicism. J. Med. Genet. 41, 289-92 
(2004).

36. Alberry, M. et al. Free fetal DNA in maternal plasma in anembryonic pregnancies: confirmation that the origin is the trophoblast. Prenat. Diagn. 27, 415-418 (2007).

37. Chen, C. et al. A pregnancy with discordant fetal and placental chromosome 18 aneuploidies revealed by invasive and noninvasive prenatal diagnosis. Reprod. Biomed. Online 29, 136-139 (2014).

38. Sharp, A. N., Heazell, A. E. P., Crocker, I. P. \& Mor, G. Placental Apoptosis in Health and Disease. Am. J. Reprod. Immunol. 64, 159-169 (2010).

39. Taglauer, E. S., Wilkins-Haug, L. \& Bianchi, D. W. Review: Cell-free fetal DNA in the maternal circulation as an indication of placental health and disease. Placenta 35, S64S68 (2014).

40. Lun, F. M. F. et al. Noninvasive prenatal methylomic analysis by genomewide bisulfite sequencing of maternal plasma DNA. Clin. Chem. 59, 1583-94 (2013).

41. Ou, X. et al. Epigenome-wide DNA methylation assay reveals placental epigenetic markers for noninvasive fetal single-nucleotide polymorphism genotyping in maternal plasma. Transfusion 54, 2523-2533 (2014).

42. Jensen, T. J. et al. Whole genome bisulfite sequencing of cell-free DNA and its cellular contributors uncovers placenta hypomethylated domains. Genome Biol. 16, 78 (2015).

43. Sun, K. et al. Plasma DNA tissue mapping by genome-wide methylation sequencing for noninvasive prenatal, cancer, and transplantation assessments. Proc. Natl. Acad. Sci. U. S. A. 112, E5503-12 (2015).

44. Walker, C. K. C. et al. Obesity and Weight Gain during Pregnancy Associate Independently with Behavior of Infant Rhesus Monkeys. Dev. Psychobiol. (2018).

45. Yin, Y. et al. Impact of cytosine methylation on DNA binding specificities of human transcription factors. Science 356, eaaj2239 (2017).

46. Dunaway, K. W. et al. Cumulative Impact of Polychlorinated Biphenyl and Large Chromosomal Duplications on DNA Methylation, Chromatin, and Expression of Autism Candidate Genes. Cell Rep. 17, 3035-3048 (2016).

47. Laufer, B. I., Hwang, H., Vogel Ciernia, A., Mordaunt, C. E. \& LaSalle, J. M. Whole genome bisulfite sequencing of Down syndrome brain reveals regional DNA hypermethylation and novel disorder insights. Epigenetics 14, 672-684 (2019).

48. Vogel Ciernia, A. et al. Epigenomic Convergence of Neural-Immune Risk Factors in Neurodevelopmental Disorder Cortex. Cereb. Cortex 30, 640-655 (2020).

49. Ducker, G. S. \& Rabinowitz, J. D. One-Carbon Metabolism in Health and Disease. Cell Metab. 25, 27-42 (2017).

50. Akram, M. Citric Acid Cycle and Role of its Intermediates in Metabolism. Cell Biochem. Biophys. 68, 475-478 (2014).

51. Horder, J. et al. Glutamate and GABA in autism spectrum disorder-a translational magnetic resonance spectroscopy study in man and rodent models. Transl. Psychiatry 8 , 106 (2018).

52. Azmitia, E. C., Saccomano, Z. T., Alzoobaee, M. F., Boldrini, M. \& Whitaker-Azmitia, P. M. Persistent Angiogenesis in the Autism Brain: An Immunocytochemical Study of Postmortem Cortex, Brainstem and Cerebellum. J. Autism Dev. Disord. 46, 1307-1318 (2016). 
53. Lilja, J. \& Ivaska, J. Integrin activity in neuronal connectivity. J. Cell Sci. 131, (2018).

54. LoParo, D. \& Waldman, I. D. The oxytocin receptor gene (OXTR) is associated with autism spectrum disorder: a meta-analysis. Mol. Psychiatry 20, 640-646 (2015).

55. Guastella, A. J. \& Hickie, I. B. Oxytocin Treatment, Circuitry, and Autism: A Critical Review of the Literature Placing Oxytocin into the Autism Context. Biol. Psychiatry 79, 234-242 (2016).

56. Kageyama, R., Ohtsuka, T. \& Kobayashi, T. The Hes gene family: repressors and oscillators that orchestrate embryogenesis. Development 134, 1243-1251 (2007).

57. Kageyama, R., Ohtsuka, T. \& Kobayashi, T. Roles of Hes genes in neural development. Dev. Growth Differ. 50 Suppl 1, S97-103 (2008).

58. Kamakura, S. et al. Hes binding to STAT3 mediates crosstalk between Notch and JAKSTAT signalling. Nat. Cell Biol. 6, 547-554 (2004).

59. Bansal, R., You, S. H., Herzig, C. T. A. \& Zoeller, R. T. Maternal thyroid hormone increases $\mathrm{HES}$ expression in the fetal rat brain: An effect mimicked by exposure to a mixture of polychlorinated biphenyls (PCBs). Dev. Brain Res. 156, 13-22 (2005).

60. Eckert, D., Buhl, S., Weber, S., Jäger, R. \& Schorle, H. The AP-2 family of transcription factors. Genome Biol. 6, 246 (2005).

61. Kuckenberg, P., Kubaczka, C. \& Schorle, H. The role of transcription factor Tcfap2c/TFAP2C in trophectoderm development. Reprod. Biomed. Online 25, 12-20 (2012).

62. Rothstein, M. \& Simoes-Costa, M. Heterodimerization of TFAP2 pioneer factors drives epigenomic remodeling during neural crest specification. Genome Res. 30, 35-48 (2020).

63. Tili, E. et al. Resveratrol decreases the levels of miR-155 by upregulating miR-663, a microRNA targeting JunB and JunD. Carcinogenesis 31, 1561-1566 (2010).

64. Pan, J. et al. Tumor-suppressive mir-663 gene induces mitotic catastrophe growth arrest in human gastric cancer cells. Oncol Rep 24, 105-112 (2010).

65. Vislovukh, A. et al. Proto-oncogenic isoform A2 of eukaryotic translation elongation factor eEF1 is a target of miR-663 and miR-744. Br. J. Cancer 108, 2304-2311 (2013).

66. Shi, C. et al. Adipogenic miRNA and meta-signature miRNAs involved in human adipocyte differentiation and obesity. Oncotarget 7, 40830-40845 (2016).

67. Michaille, J.-J. et al. MiR-663, a MicroRNA Linked with Inflammation and Cancer That Is under the Influence of Resveratrol. Med. (Basel, Switzerland) 5, (2018).

68. Greco, A., Goossens, R., van Engelen, B. \& van der Maarel, S. M. Consequences of epigenetic derepression in facioscapulohumeral muscular dystrophy. Clin. Genet. 97, 799-814 (2020).

69. van Overveld, P. G. M. et al. Hypomethylation of D4Z4 in 4q-linked and non-4q-linked facioscapulohumeral muscular dystrophy. Nat. Genet. 35, 315-317 (2003).

70. Himeda, C. L., Jones, T. I. \& Jones, P. L. Targeted epigenetic repression by CRISPR/dSaCas9 suppresses pathogenic DUX4-fl expression in FSHD. Mol. Ther. Methods Clin. Dev. 20, 298-311 (2021).

71. Ledonne, A. et al. Neuregulin $1 /$ ErbB signalling modulates hippocampal mGluRIdependent LTD and object recognition memory. Pharmacol. Res. 130, 12-24 (2018).

72. Yan, Y. et al. Omega-3 fatty acids prevent inflammation and metabolic disorder through inhibition of NLRP3 inflammasome activation. Immunity 38, 1154-1163 (2013). 
73. Hilgendorf, K. I. et al. Omega-3 Fatty Acids Activate Ciliary FFAR4 to Control Adipogenesis. Cell 179, 1289-1305.e21 (2019).

74. Dehaut, F., Bertrand, I., Miltaud, T., Pouplard-Barthelaix, A. \& Maingault, M. n-6 polyunsaturated fatty acids increase the neurite length of PC12 cells and embryonic chick motoneurons. Neurosci. Lett. 161, 133-136 (1993).

75. Kamata, Y., Shiraga, H., Tai, A., Kawamoto, Y. \& Gohda, E. Induction of neurite outgrowth in PC12 cells by the medium-chain fatty acid octanoic acid. Neuroscience 146, 10731081 (2007).

76. Hennebelle, M. et al. Linoleic acid-derived metabolites constitute the majority of oxylipins in the rat pup brain and stimulate axonal growth in primary rat cortical neuronglia co-cultures in a sex-dependent manner. J. Neurochem. 152, 195-207 (2020).

77. Jory, J. Abnormal fatty acids in Canadian children with autism. Nutrition 32, 474-477 (2016).

78. Alfadhel, M. et al. Asparagine synthetase deficiency: New inborn errors of metabolism. in JIMD Reports (eds. Zschocke, J. et al.) 22, 11-16 (Springer Berlin Heidelberg, 2015).

79. C Yuen, R. K. et al. Whole genome sequencing resource identifies 18 new candidate genes for autism spectrum disorder. Nat. Neurosci. 20, 602-611 (2017).

80. Smith, S. R. et al. Agouti expression in human adipose tissue: functional consequences and increased expression in type 2 diabetes. Diabetes 52, 2914-2922 (2003).

81. Christiansen, T., Richelsen, B. \& Bruun, J. M. Monocyte chemoattractant protein-1 is produced in isolated adipocytes, associated with adiposity and reduced after weight loss in morbid obese subjects. Int. J. Obes. (Lond). 29, 146-150 (2005).

82. Azar Sharabiani, M. T. et al. Immunologic profile of excessive body weight. Biomarkers 16, 243-251 (2011).

83. Brown, A. S. et al. Elevated maternal C-reactive protein and autism in a national birth cohort. Mol. Psychiatry 19, 259-264 (2014).

84. Zerbo, O. et al. Maternal mid-pregnancy C-reactive protein and risk of autism spectrum disorders: the early markers for autism study. Transl. Psychiatry 6, e783 (2016).

85. Madan, J. C. et al. Maternal obesity and markers of inflammation in pregnancy. Cytokine 47, 61-64 (2009).

86. Summers, L., Clingerman, K. J. \& Yang, X. Validation of a body condition scoring system in rhesus macaques (Macaca mulatta): assessment of body composition by using dualenergy X-ray absorptiometry. J. Am. Assoc. Lab. Anim. Sci. 51, 88-93 (2012).

87. Smith, S. Infant cross-fostering in rhesus monkeys (Macaca mulatta): A procedure for the long-term management of captive populations. Am. J. Primatol. 11, 229-237 (1986).

88. Golub, M. S., Hogrefe, C. E. \& VandeVoort, C. A. Binge Drinking Prior to Pregnancy Detection in a Nonhuman Primate: Behavioral Evaluation of Offspring. Alcohol. Clin. Exp. Res. 38, 551-556 (2014).

89. Sclafani, V. et al. Early Predictors of Impaired Social Functioning in Male Rhesus Macaques (Macaca mulatta). PLoS One 11, e0165401 (2016).

90. Hasegawa, Y. et al. Optimization of a Method for the Simultaneous Extraction of Polar and Non-Polar Oxylipin Metabolites, DNA, RNA, Small RNA, and Protein from a Single Small Tissue Sample. Methods Protoc. 3, (2020).

91. Ichihara, K. \& Fukubayashi, Y. Preparation of fatty acid methyl esters for gas-liquid 
chromatography. J. Lipid Res. 51, 635-640 (2010).

92. Meier, S., Mjøs, S. A., Joensen, H. \& Grahl-Nielsen, O. Validation of a one-step extraction/methylation method for determination of fatty acids and cholesterol in marine tissues. J. Chromatogr. A 1104, 291-298 (2006).

93. Zhang, Z., Richardson, C. E., Hennebelle, M. \& Taha, A. Y. Validation of a One-Step Method for Extracting Fatty Acids from Salmon, Chicken and Beef Samples. J. Food Sci. 82, 2291-2297 (2017).

94. Li, H. et al. The Sequence Alignment/Map format and SAMtools. Bioinformatics 25, 20782079 (2009).

95. Martin, M. Cutadapt removes adapter sequences from high-throughput sequencing reads. EMBnet.journal 17, 10 (2011).

96. Wingett, S. W. \& Andrews, S. FastQ Screen: A tool for multi-genome mapping and quality control. F1000Research 7, 1338 (2018).

97. Krueger, F. \& Andrews, S. R. Bismark: a flexible aligner and methylation caller for Bisulfite-Seq applications. Bioinformatics 27, 1571-1572 (2011).

98. Langmead, B. \& Salzberg, S. L. Fast gapped-read alignment with Bowtie 2. Nat. Methods 9, 357-359 (2012).

99. Ewels, P., Magnusson, M., Lundin, S. \& Käller, M. MultiQC: summarize analysis results for multiple tools and samples in a single report. Bioinformatics 32, 3047-3048 (2016).

100. Laufer, B. I. et al. Low-pass whole genome bisulfite sequencing of neonatal dried blood spots identifies a role for RUNX1 in down syndrome DNA methylation profiles. Hum. Mol. Genet. 29, 3465-3476 (2020).

101. Korthauer, K., Chakraborty, S., Benjamini, Y. \& Irizarry, R. A. Detection and accurate false discovery rate control of differentially methylated regions from whole genome bisulfite sequencing. Biostatistics 20, 367-383 (2018).

102. Hansen, K. D., Langmead, B. \& Irizarry, R. A. BSmooth: from whole genome bisulfite sequencing reads to differentially methylated regions. Genome Biol. 13, R83 (2012).

103. Yu, G., Wang, L.-G. \& He, Q.-Y. ChIPseeker: an R/Bioconductor package for ChIP peak annotation, comparison and visualization. Bioinformatics 31, 2382-2383 (2015).

104. Rainer, J., Gatto, L. \& Weichenberger, C. X. ensembldb: an R package to create and use Ensembl-based annotation resources. Bioinformatics 35, 3151-3153 (2019).

105. Lex, A., Gehlenborg, N., Strobelt, H., Vuillemot, R. \& Pfister, H. UpSet: Visualization of Intersecting Sets, IEEE Trans. Vis. Comput. Graph. 20, 1983-1992 (2014).

106. Conway, J. R., Lex, A. \& Gehlenborg, N. UpSetR: an R package for the visualization of intersecting sets and their properties. Bioinformatics 33, 2938-2940 (2017).

107. Krassowski, M. ComplexUpset. (2020). doi:10.5281/zenodo.3700590

108. Prüfer, K. et al. FUNC: A package for detecting significant associations between gene sets and ontological annotations. BMC Bioinformatics 8, 41 (2007).

109. Grote, S. GOfuncR: Gene ontology enrichment using FUNC. (2020).

110. Sayols, S. rrvgo: a Bioconductor package to reduce and visualize Gene Ontology terms. (2020).

111. Chen, E. Y. et al. Enrichr: interactive and collaborative HTML5 gene list enrichment analysis tool. BMC Bioinformatics 14, 128 (2013).

112. Kuleshov, M. V. et al. Enrichr: a comprehensive gene set enrichment analysis web server 
2016 update. Nucleic Acids Res. 44, W90-W97 (2016).

113. Xie, Z. et al. Gene Set Knowledge Discovery with Enrichr. Curr. Protoc. 1, e90 (2021).

114. Jawaid, W. enrichR: Provides an R Interface to 'Enrichr'. (2021).

115. Gel, B. et al. regioneR: An R/Bioconductor package for the association analysis of genomic regions based on permutation tests. Bioinformatics 32, 289-291 (2016).

116. Heger, A., Webber, C., Goodson, M., Ponting, C. P. \& Lunter, G. GAT: a simulation framework for testing the association of genomic intervals. Bioinformatics 29, 2046-2048 (2013).

117. Bailey, T. L. et al. MEME Suite: tools for motif discovery and searching. Nucleic Acids Res. 37, W202-W208 (2009).

118. McLeay, R. C. \& Bailey, T. L. Motif Enrichment Analysis: a unified framework and an evaluation on ChIP data. BMC Bioinformatics 11, 165 (2010).

119. Nystrom, S. memes: motif matching, comparison, and de novo discovery using the MEME Suite. (2021).

120. Langfelder, P. \& Horvath, S. WGCNA: an R package for weighted correlation network analysis. BMC Bioinformatics 9, 559 (2008).

121. Song, L., Langfelder, P. \& Horvath, S. Comparison of co-expression measures: mutual information, correlation, and model based indices. BMC Bioinformatics 13, 328 (2012). 


\section{Experimental Timeline}

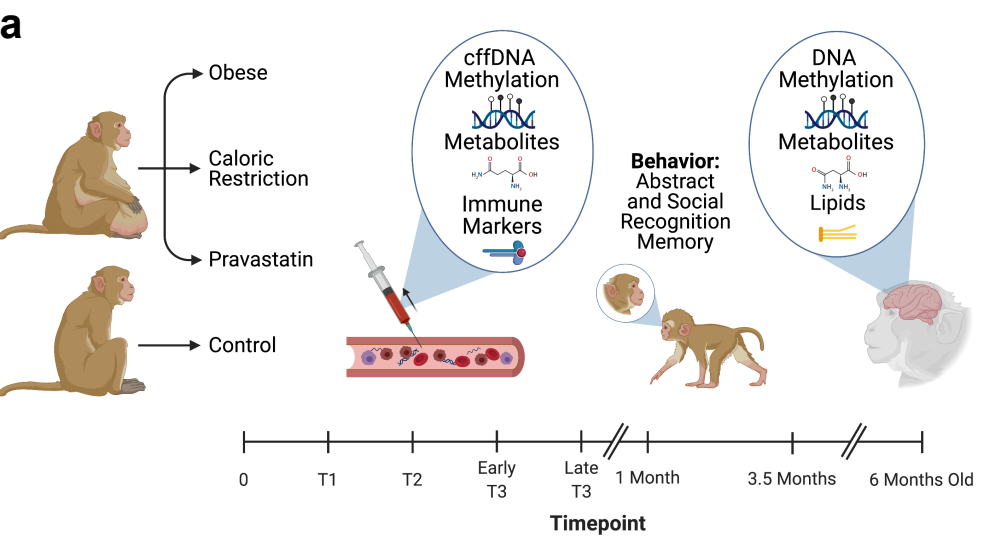

Caloric Restriction vs. Obese Gene Overlaps
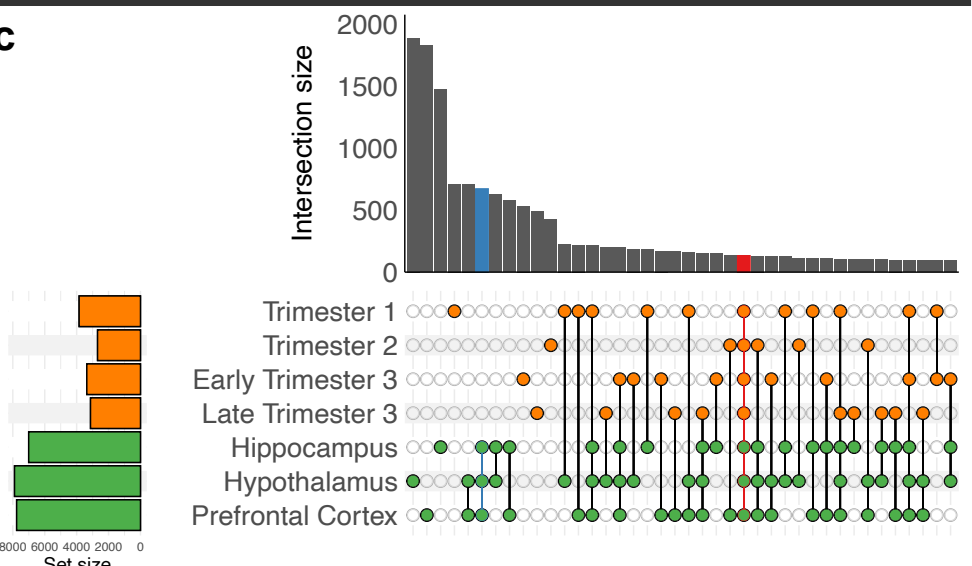

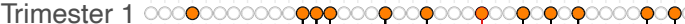
Trimester 200000000000 Early Trimester 3000000000 Late Trimester 3 0000000000

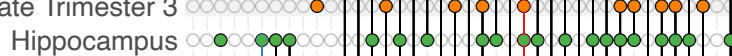

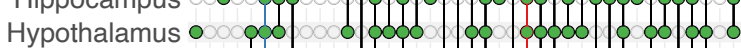

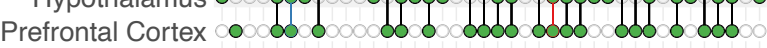

Obese vs. Control Gene Overlaps
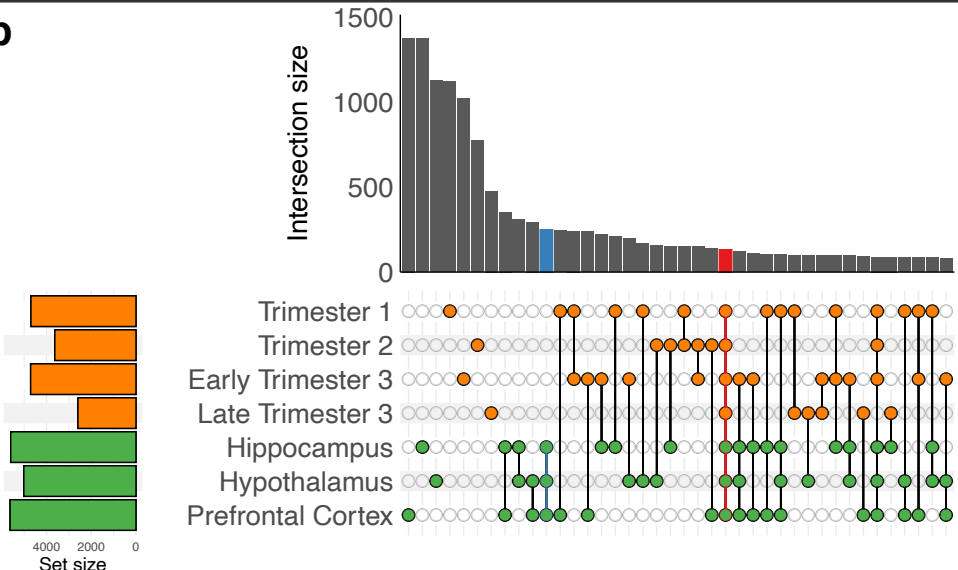

Trimester 1000000000000900090000000 (P) Trimester 2000000

Early Trimester 30000000000 Late Trimester 3 o000000000

Hippocampus co0000opo oo

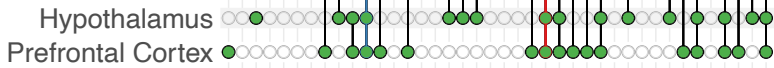

\section{Pravastatin vs. Obese Gene Overlaps}

\section{d}

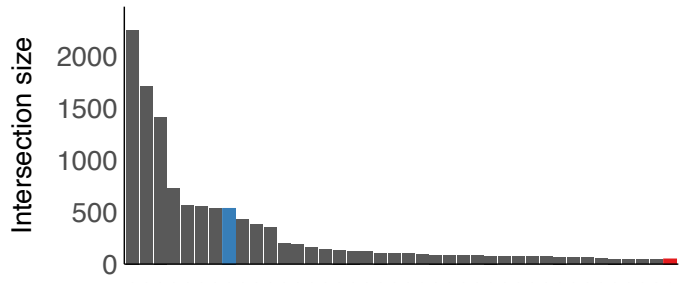

Trimester 10000000000000000000000000000000000000000

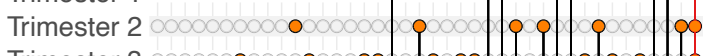

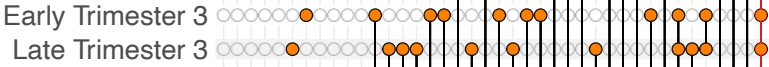

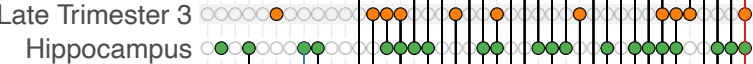

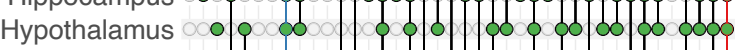

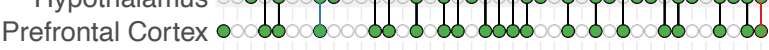


Anatomical structure morphogenesis Nervous system development Anatomical structure development Plasma membrane bounded cell projection organization Cell adhesion Movement of cell or subcellular component Multicellular organismal process Cell projection Plasma membrane Cell junction Plasma membrane region Intrinsic component of plasma membrane

$$
\text { Dendritic tree }
$$

Receptor complex Anion binding

Cytoskeletal protein binding

Gated channel activity

GTPase activator activity Carbohydrate derivative binding Extracellular matrix structural constituent Transmembrane receptor protein kinase activity

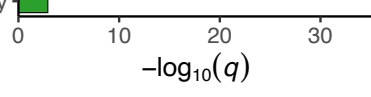

Gene Ontology $\square$ Biological Process

Cellular Component

b

Nervous system development cell projection organization Cell junction organization

Cell morphogenesis

Synaptic signaling

Anatomical structure development Enzyme linked receptor protein signaling pathway

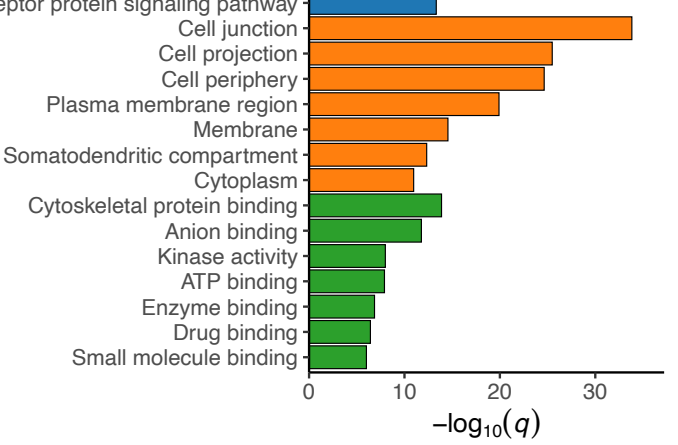

Integrin signallng pathway

Endothelin signaling pathway Ionotropic glutamate receptor pathway

PDGF signaling pathway

$$
\text { Angiogenesis }
$$

CCKR signaling map ST

Metabotropic glutamate receptor group III pathway EGF receptor signaling pathway Histamine $\mathrm{H} 1$ receptor mediated signaling pathway

Heterotrimeric $\mathrm{G}$-protein signaling pathway-Gi

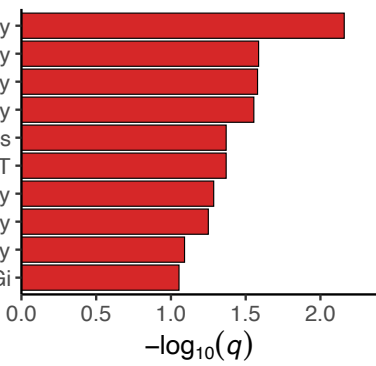

d Thyrotropin-releasing hormone receptor signalining

Oxytocin receptor mediated signaling pathway Ionotropic glutamate receptor pathway

5 HT2 type receptor mediated signaling pathway Angiogenesis

Muscarinic acetylcholine receptor 1 and 3 signaling Integrin signaling pathway

EGF receptor signaling pathway Histamine $\mathrm{H} 1$ receptor mediated signaling pathway PDGF signaling pathway

$$
0
$$

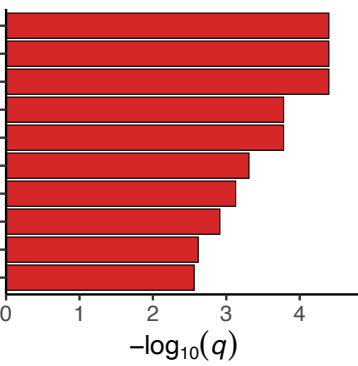

HES2-eDBD-methyl-1 HES1-eDBD-methyl-1 TFAP2C-eDBD-1 TBX2-eDBD-methyl-1 TBX2-eDBD-1 HES5-eDBD-methyl-2 IRX1-eDBD-methyl-1 ELK4-FL-methyl-1 BATF-FL-1 HEY1-FL-methyl-1

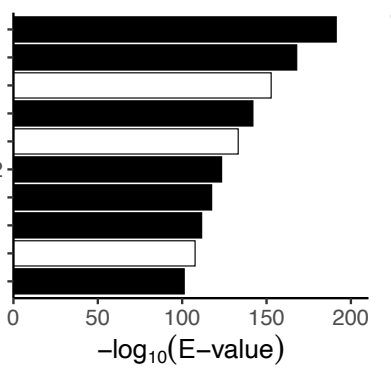

f
Not Methylated

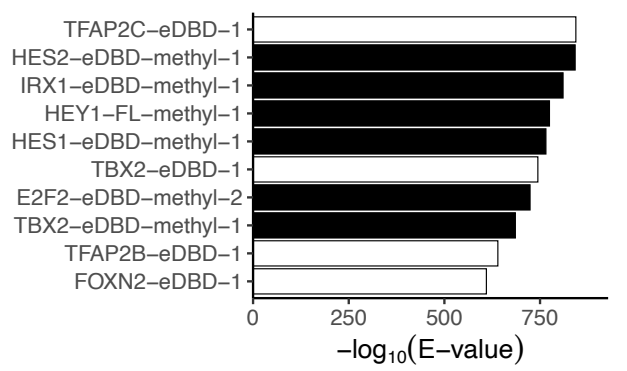




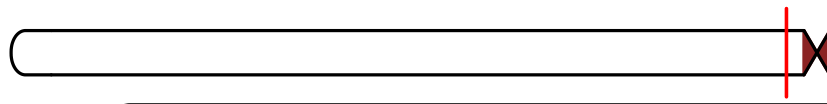

cffDNA
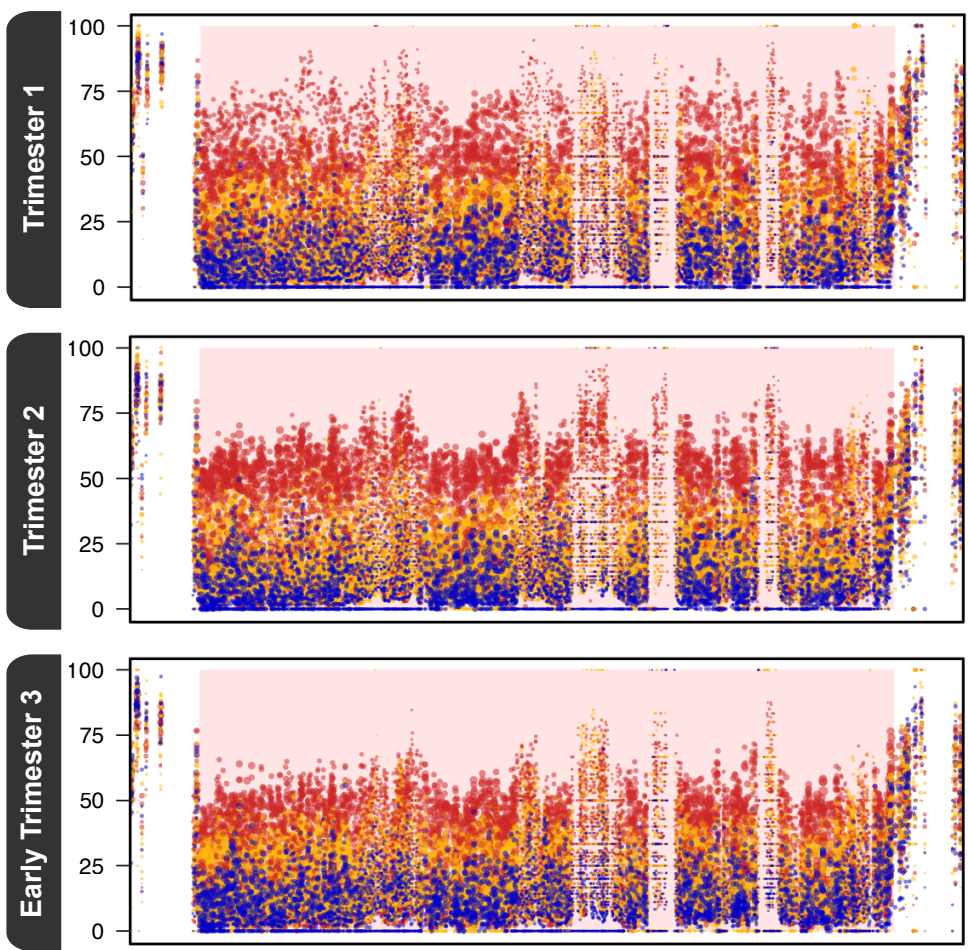

m 100

$\begin{aligned} & 100 \\ & 75 \\ & 50 \\ & 25 \\ & 0\end{aligned}-$

Obese
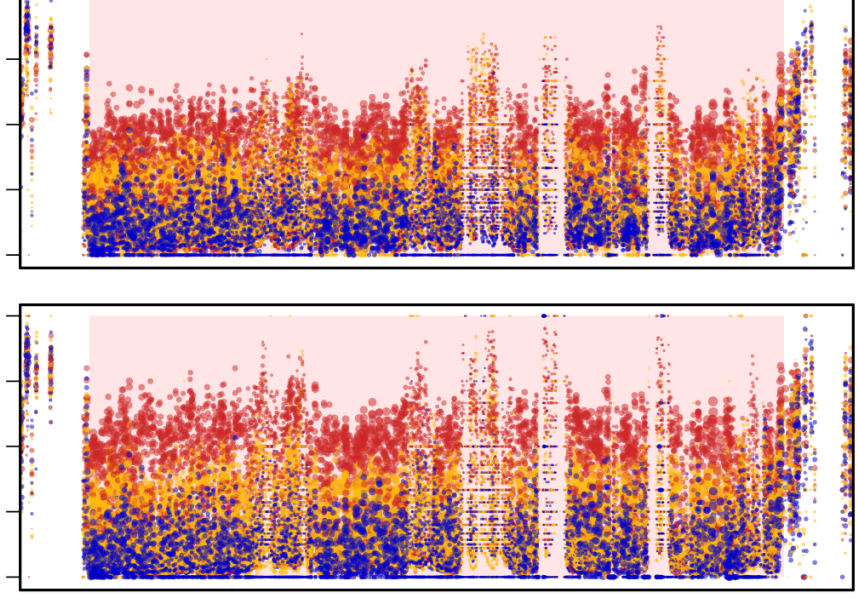

Intervention

Control

\section{Brain}
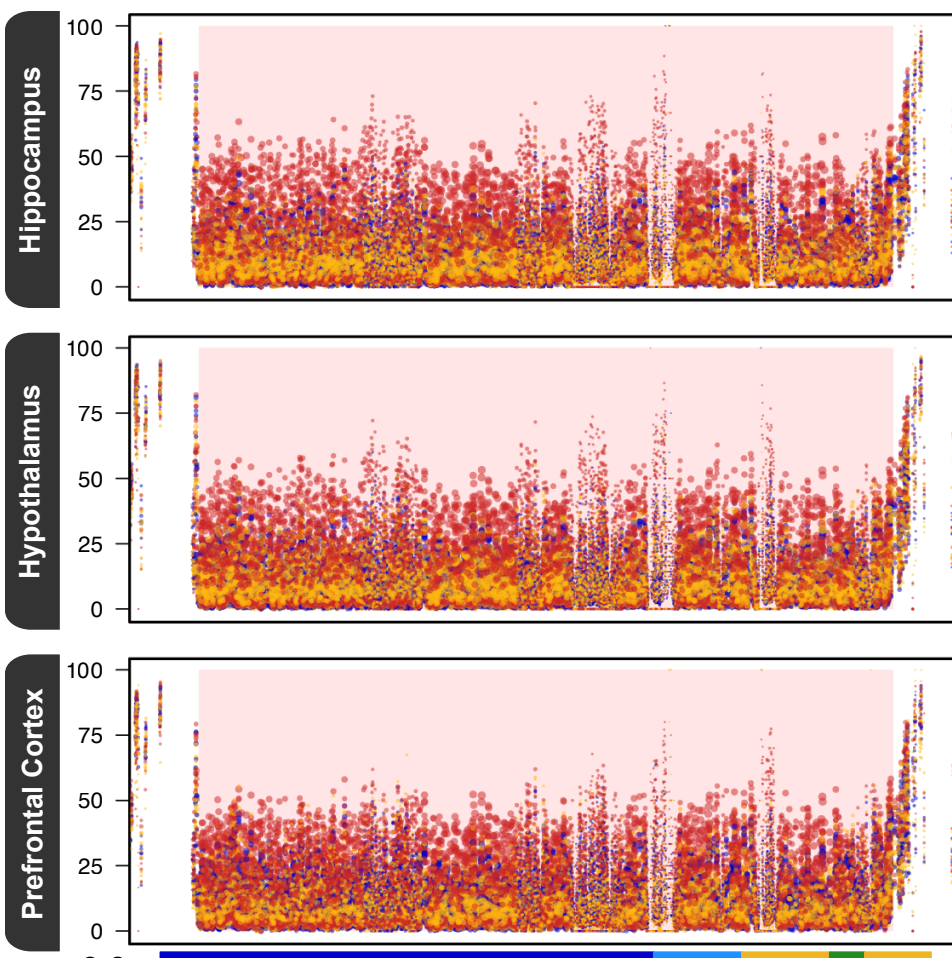

$\begin{aligned} & 100 \\ & 75 \\ & 50 \\ & 25 \\ & 0\end{aligned}-$

CpGs
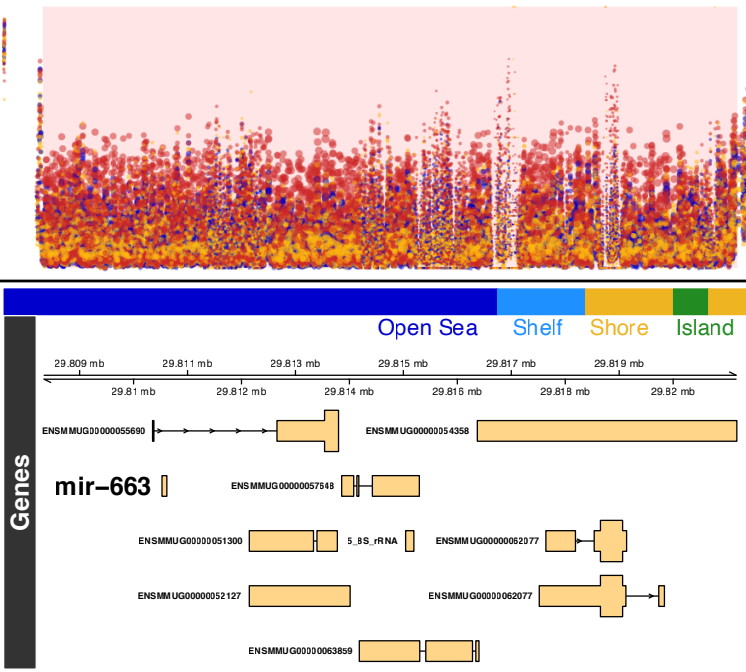

b

\begin{tabular}{|c|c|c|c|c|c|c|c|c|c|c|c|c|c|c|c|c|c|c|c|c|c|c|c|c|}
\hline \multicolumn{4}{|c|}{ Maternal Group } & \multicolumn{2}{|c|}{ Behavior } & \multicolumn{11}{|c|}{ Immune Markers } & \multicolumn{8}{|c|}{ Metabolomics } \\
\hline $\begin{array}{c}0.39 \\
(0.05)\end{array}$ & $\begin{array}{c}-0.29 \\
(0.2)\end{array}$ & $\begin{array}{c}0.1 \\
(0.6)\end{array}$ & $\begin{array}{l}-0.24 \\
(0.2)\end{array}$ & $\begin{array}{c}-0.25 \\
(0.2)\end{array}$ & $\begin{array}{c}0.34 \\
(0.09)\end{array}$ & \begin{tabular}{|c}
-0.079 \\
$(0.7)$
\end{tabular} & $\begin{array}{c}0.41 \\
(0.04)\end{array}$ & $\begin{array}{c}0.41 \\
(0.04)\end{array}$ & $\begin{array}{c}-0.14 \\
(0.5)\end{array}$ & $\begin{array}{c}-0.056 \\
(0.8)\end{array}$ & $\begin{array}{c}0.43 \\
(0.03)\end{array}$ & $\begin{array}{l}0.28 \\
(0.2)\end{array}$ & $\begin{array}{c}0.46 \\
(0.02)\end{array}$ & $\begin{array}{c}0.59 \\
(0.002)\end{array}$ & $\begin{array}{l}0.12 \\
(0.6)\end{array}$ & $\begin{array}{c}0.079 \\
(0.7)\end{array}$ & $\begin{array}{c}0.55 \\
(0.004)\end{array}$ & $\begin{array}{c}0.65 \\
(4 e-04)\end{array}$ & $\begin{array}{c}-0.62 \\
(9 e-04)\end{array}$ & $\begin{array}{c}0.47 \\
(0.02)\end{array}$ & $\begin{array}{c}0.4 \\
(0.05)\end{array}$ & $\begin{array}{c}-0.011 \\
(1)\end{array}$ & $\begin{array}{c}0.44 \\
(0.03)\end{array}$ & $\begin{array}{l}0.16 \\
(0.5)\end{array}$ \\
\hline $\begin{array}{c}0.42 \\
(0.04)\end{array}$ & $\begin{array}{c}-0.28 \\
(0.2)\end{array}$ & $\begin{array}{c}0.011 \\
(1)\end{array}$ & $\begin{array}{l}-0.19 \\
(0.4)\end{array}$ & $\begin{array}{l}-0.23 \\
(0.3)\end{array}$ & $\begin{array}{l}0.31 \\
(0.1)\end{array}$ & \begin{tabular}{|c|}
-0.017 \\
$(0.9)$
\end{tabular} & $\begin{array}{c}-0.11 \\
(0.6)\end{array}$ & $\begin{array}{c}-0.14 \\
(0.5)\end{array}$ & $\begin{array}{c}0.42 \\
(0.04)\end{array}$ & $\begin{array}{c}0.6 \\
(0.001)\end{array}$ & $\begin{array}{c}0.47 \\
(0.02)\end{array}$ & $\begin{array}{l}0.039 \\
(0.9)\end{array}$ & $\begin{array}{c}-0.074 \\
(0.7)\end{array}$ & $\begin{array}{c}-0.045 \\
(0.8)\end{array}$ & $\begin{array}{c}0.4 \\
(0.05)\end{array}$ & $\begin{array}{c}0.5 \\
(0.01)\end{array}$ & $\begin{array}{l}0.11 \\
(0.6)\end{array}$ & $\begin{array}{c}0.042 \\
(0.8)\end{array}$ & $\begin{array}{l}-0.34 \\
(0.09)\end{array}$ & $\begin{array}{l}0.14 \\
(0.5)\end{array}$ & $\begin{array}{c}-0.14 \\
(0.5)\end{array}$ & $\begin{array}{l}-0.46 \\
(0.02)\end{array}$ & $\begin{array}{c}-0.022 \\
(0.9)\end{array}$ & $\begin{array}{c}0.053 \\
(0.8)\end{array}$ \\
\hline $\begin{array}{c}0.41 \\
(0.04)\end{array}$ & $\begin{array}{c}-0.29 \\
(0.2)\end{array}$ & $\begin{array}{c}0.0057 \\
(1)\end{array}$ & $\begin{array}{c}-0.15 \\
(0.5)\end{array}$ & $\begin{array}{l}-0.21 \\
(0.3)\end{array}$ & $\begin{array}{l}0.28 \\
(0.2)\end{array}$ & $\begin{array}{l}0.039 \\
(0.9)\end{array}$ & $\begin{array}{c}-0.049 \\
(0.8)\end{array}$ & $\begin{array}{c}-0.11 \\
(0.6)\end{array}$ & $\begin{array}{c}-0.0023 \\
\text { (1) }\end{array}$ & $\begin{array}{ll}3 & 0.13 \\
& (0.6)\end{array}$ & $\begin{array}{l}0.17 \\
(0.4)\end{array}$ & $\begin{array}{l}0.12 \\
(0.6)\end{array}$ & $\begin{array}{c}0.0078 \\
(1)\end{array}$ & $\begin{array}{l}0.32 \\
(0.1)\end{array}$ & $\begin{array}{l}0.11 \\
(0.6)\end{array}$ & $\begin{array}{l}0.11 \\
(0.6)\end{array}$ & \begin{tabular}{|c|}
0.46 \\
$(0.02)$
\end{tabular} & $\begin{array}{c}0.0011 \\
(1)\end{array}$ & $\begin{array}{c}-0.085 \\
(0.7)\end{array}$ & $\begin{array}{l}0.018 \\
(0.9)\end{array}$ & $\begin{array}{c}-0.26 \\
(0.2)\end{array}$ & $\begin{array}{l}-0.3 \\
(0.1)\end{array}$ & $\begin{array}{c}-0.023 \\
(0.9)\end{array}$ & $\begin{array}{c}0.34 \\
(0.09)\end{array}$ \\
\hline $\begin{array}{c}0.44 \\
(0.03)\end{array}$ & $\begin{array}{l}-0.31 \\
(0.1)\end{array}$ & $\begin{array}{c}-0.0061 \\
\text { (1) }\end{array}$ & $\begin{array}{c}-0.14 \\
(0.5)\end{array}$ & $\begin{array}{c}-0.29 \\
(0.2)\end{array}$ & $\begin{array}{l}0.26 \\
(0.2)\end{array}$ & $\begin{array}{c}0.42 \\
(0.04)\end{array}$ & $\begin{array}{l}0.11 \\
(0.6)\end{array}$ & $\begin{array}{c}0.39 \\
(0.05)\end{array}$ & $\begin{array}{l}0.035 \\
(0.9)\end{array}$ & $\begin{array}{l}0.16 \\
(0.4)\end{array}$ & $\begin{array}{c}0.36 \\
(0.08)\end{array}$ & $\begin{array}{c}0.36 \\
(0.08)\end{array}$ & $\begin{array}{c}0.36 \\
(0.07)\end{array}$ & $\begin{array}{c}0.44 \\
(0.03)\end{array}$ & $\begin{array}{c}0.2 \\
(0.3)\end{array}$ & $\begin{array}{l}0.17 \\
(0.4)\end{array}$ & $\begin{array}{c}0.38 \\
(0.06)\end{array}$ & $\begin{array}{c}-0.039 \\
(0.9)\end{array}$ & $\begin{array}{c}-0.25 \\
(0.2)\end{array}$ & $\begin{array}{l}0.23 \\
(0.3)\end{array}$ & $\begin{array}{c}0.028 \\
(0.9)\end{array}$ & $\begin{array}{l}-0.13 \\
(0.5)\end{array}$ & $\begin{array}{c}-0.15 \\
(0.5)\end{array}$ & $\begin{array}{c}0.47 \\
(0.02)\end{array}$ \\
\hline
\end{tabular}

C

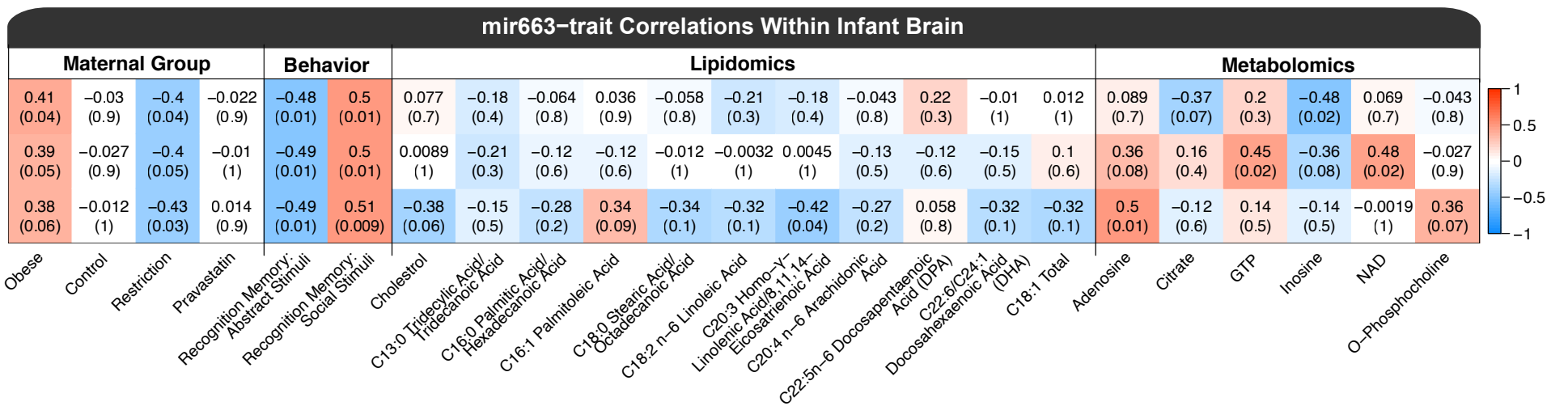




\begin{tabular}{|c|c|c|c|c|c|c|c|c|c|c|c|c|c|c|c|c|c|c|c|}
\hline \multirow[b]{2}{*}{ Green } & \multicolumn{4}{|c|}{ Maternal Group } & \multicolumn{2}{|c|}{ Behavior } & \multicolumn{6}{|c|}{ Lipidomics } & \multicolumn{7}{|c|}{ Metabolomics } \\
\hline & $\begin{array}{l}0.15 \\
(0.5)\end{array}$ & $\begin{array}{c}0.41 \\
(0.04)\end{array}$ & $\begin{array}{l}-0.49 \\
(0.01)\end{array}$ & $\begin{array}{c}-0.11 \\
(0.6)\end{array}$ & $\begin{array}{c}-0.17 \\
(0.4)\end{array}$ & $\begin{array}{l}0.12 \\
(0.6)\end{array}$ & $\begin{array}{l}0.17 \\
(0.4)\end{array}$ & $\begin{array}{l}0.22 \\
(0.3)\end{array}$ & $\begin{array}{l}0.12 \\
(0.6)\end{array}$ & $\begin{array}{l}0.13 \\
(0.5)\end{array}$ & $\begin{array}{l}0.14 \\
(0.5)\end{array}$ & $\begin{array}{l}0.18 \\
(0.4)\end{array}$ & $\begin{array}{c}-0.5 \\
(0.01)\end{array}$ & $\begin{array}{c}-0.19 \\
(0.4)\end{array}$ & $\begin{array}{c}0.006 \\
(1)\end{array}$ & $\begin{array}{c}-0.088 \\
(0.7)\end{array}$ & $\begin{array}{c}-0.042 \\
(0.8)\end{array}$ & $\begin{array}{c}-0.19 \\
(0.4)\end{array}$ & $\begin{array}{l}0.14 \\
(0.5)\end{array}$ \\
\hline Yellow & $\begin{array}{r}-0.42 \\
(0.04) \\
\end{array}$ & $\begin{array}{l}0.18 \\
(0.4) \\
\end{array}$ & $\begin{array}{c}-0.29 \\
(0.2) \\
\end{array}$ & $\begin{array}{c}0.51 \\
(0.01) \\
\end{array}$ & $\begin{array}{c}-0.013 \\
(0.9)\end{array}$ & $\begin{array}{r}-0.36 \\
(0.08) \\
\end{array}$ & $\begin{array}{c}-0.21 \\
(0.3) \\
\end{array}$ & $\begin{array}{c}-0.16 \\
(0.4) \\
\end{array}$ & $\begin{array}{l}-0.1 \\
(0.6) \\
\end{array}$ & $\begin{array}{c}-0.045 \\
(0.8)\end{array}$ & $\begin{array}{c}-0.31 \\
(0.1) \\
\end{array}$ & $\begin{array}{c}-0.33 \\
(0.1) \\
\end{array}$ & $\begin{array}{c}-0.22 \\
(0.3) \\
\end{array}$ & $\begin{array}{c}0.053 \\
(0.8) \\
\end{array}$ & $\begin{array}{l}0.21 \\
(0.3) \\
\end{array}$ & $\begin{array}{c}-0.13 \\
(0.5)\end{array}$ & $\begin{array}{c}-0.12 \\
(0.6) \\
\end{array}$ & $\begin{array}{c}-0.11 \\
(0.6) \\
\end{array}$ & $\begin{array}{c}-0.052 \\
(0.8) \\
\end{array}$ \\
\hline Blue & $\begin{array}{l}-0.45 \\
(0.02)\end{array}$ & $\begin{array}{c}0.35 \\
(0.08)\end{array}$ & $\begin{array}{l}0.18 \\
(0.4)\end{array}$ & $\begin{array}{c}-0.045 \\
(0.8)\end{array}$ & $\begin{array}{c}0.46 \\
(0.02)\end{array}$ & $\begin{array}{c}-0.58 \\
(0.002)\end{array}$ & $\begin{array}{c}0.38 \\
(0.06)\end{array}$ & $\begin{array}{c}0.35 \\
(0.09)\end{array}$ & $\begin{array}{c}0.48 \\
(0.01)\end{array}$ & $\begin{array}{c}0.42 \\
(0.04)\end{array}$ & $\begin{array}{l}0.34 \\
(0.1)\end{array}$ & $\begin{array}{l}0.34 \\
(0.1)\end{array}$ & $\begin{array}{l}-0.38 \\
(0.06)\end{array}$ & $\begin{array}{c}0.42 \\
(0.03)\end{array}$ & $\begin{array}{c}0.47 \\
(0.02)\end{array}$ & $\begin{array}{c}-0.33 \\
(0.1)\end{array}$ & $\begin{array}{l}0.31 \\
(0.1)\end{array}$ & $\begin{array}{c}0.38 \\
(0.06)\end{array}$ & $\begin{array}{c}-0.33 \\
(0.1)\end{array}$ \\
\hline Brown & $\begin{array}{l}-0.15 \\
(0.5)\end{array}$ & $\begin{array}{c}-0.15 \\
(0.5)\end{array}$ & $\begin{array}{l}0.19 \\
(0.4)\end{array}$ & $\begin{array}{l}0.12 \\
(0.6)\end{array}$ & $\begin{array}{l}0.15 \\
(0.5)\end{array}$ & $\begin{array}{c}-0.051 \\
(0.8)\end{array}$ & $\begin{array}{l}-0.36 \\
(0.08)\end{array}$ & $\begin{array}{c}-0.29 \\
(0.2)\end{array}$ & $\begin{array}{l}0.27 \\
(0.2)\end{array}$ & $\begin{array}{c}-0.038 \\
(0.9)\end{array}$ & $\begin{array}{l}-0.38 \\
(0.06)\end{array}$ & $\begin{array}{l}-0.3 \\
(0.1)\end{array}$ & $\begin{array}{c}0.00059 \\
\text { (1) }\end{array}$ & $\begin{array}{c}0.076 \\
(0.7)\end{array}$ & $\begin{array}{l}0.18 \\
(0.4)\end{array}$ & $\begin{array}{l}-0.2 \\
(0.3)\end{array}$ & $\begin{array}{c}-0.27 \\
(0.2)\end{array}$ & $\begin{array}{c}-0.27 \\
(0.2)\end{array}$ & $\begin{array}{l}0.024 \\
(0.9)\end{array}$ \\
\hline urquoise & $\begin{array}{c}-0.15 \\
(0.5)\end{array}$ & $\begin{array}{c}0.3 \\
(0.1)\end{array}$ & $\begin{array}{l}0.073 \\
(0.7)\end{array}$ & $\begin{array}{l}-0.2 \\
(0.3)\end{array}$ & $\begin{array}{c}0.3 \\
(0.1)\end{array}$ & $\begin{array}{l}0.11 \\
(0.6)\end{array}$ & $\begin{array}{l}0.03 \\
(0.9)\end{array}$ & $\begin{array}{l}0.066 \\
(0.8)\end{array}$ & $\begin{array}{l}0.33 \\
(0.1)\end{array}$ & $\begin{array}{l}0.31 \\
(0.1)\end{array}$ & $\begin{array}{c}-0.17 \\
(0.4)\end{array}$ & $\begin{array}{l}0.039 \\
(0.9)\end{array}$ & $\begin{array}{c}0.048 \\
(0.8)\end{array}$ & $\begin{array}{c}-0.14 \\
(0.5)\end{array}$ & $\begin{array}{l}0.14 \\
(0.5)\end{array}$ & $\begin{array}{c}0.058 \\
(0.8)\end{array}$ & $\begin{array}{c}-0.13 \\
(0.5)\end{array}$ & $\begin{array}{c}-0.24 \\
(0.3)\end{array}$ & $\begin{array}{c}-0.12 \\
(0.6)\end{array}$ \\
\hline
\end{tabular}

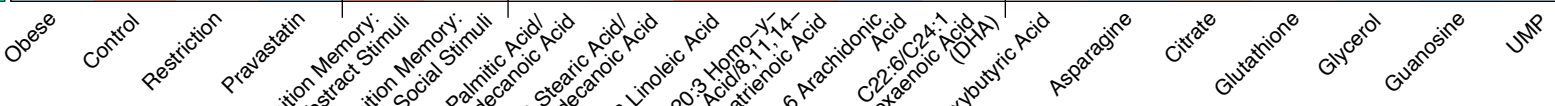

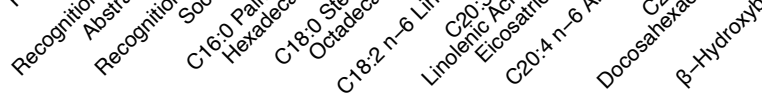

\section{Blue Module Eigengene-trait Relationships}

b
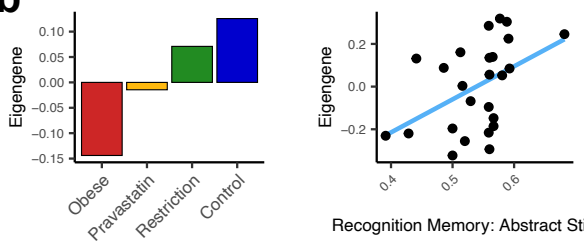

Recognition Memory: Abstract Stimul
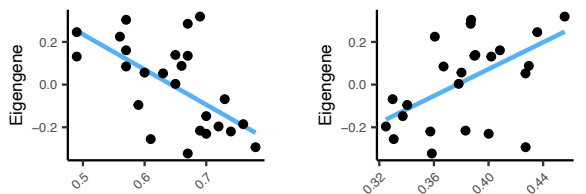

C18:2 n-6 Linoleic Acid (mg/g)

\section{Blue Module Maternal Obesity Co-methylation Network}

\section{C}

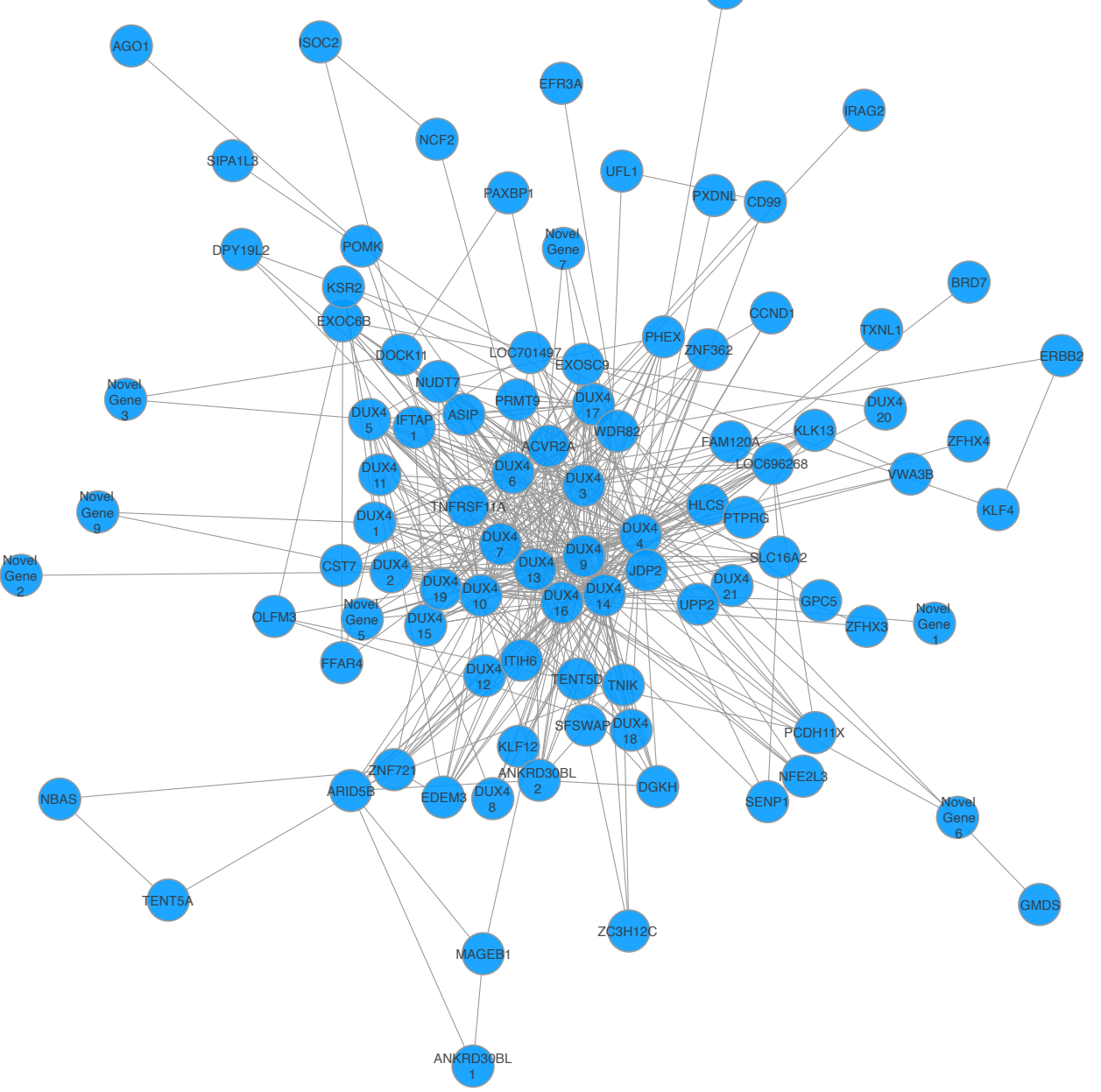

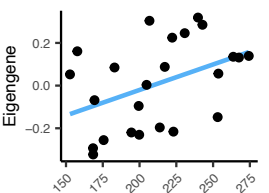

Asparagine $(\mathrm{nmol} / \mathrm{mg})$

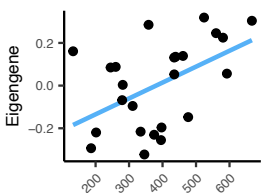

Citrate $(\mathrm{nmol} / \mathrm{mg})$

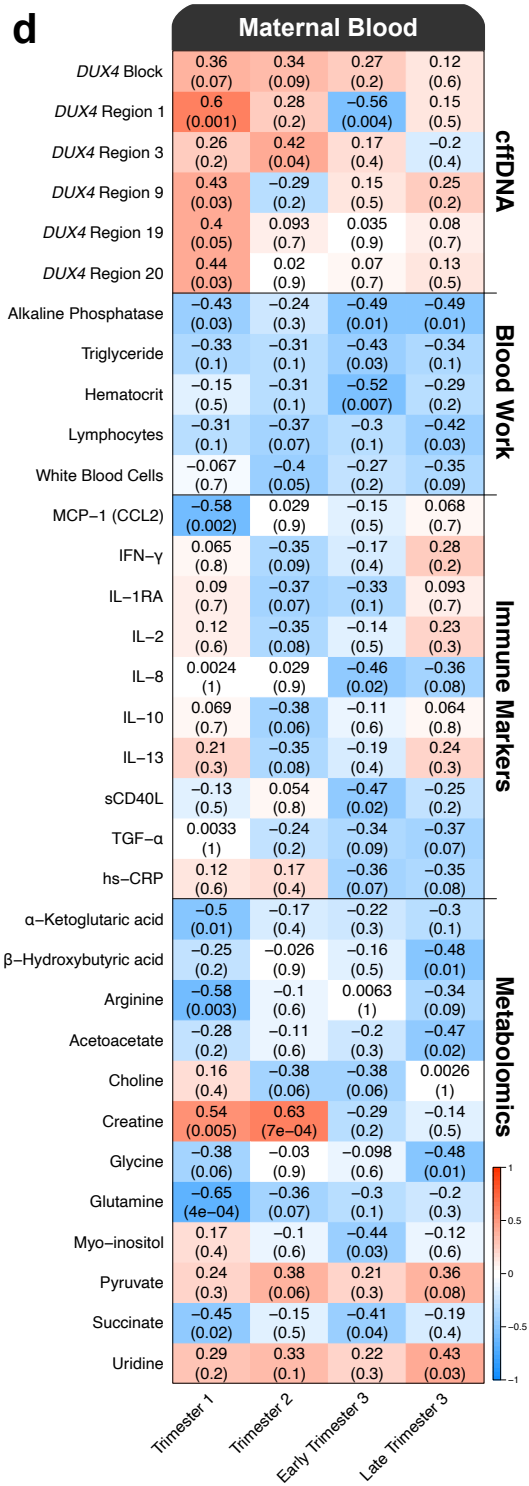

